\title{
Numerical Treatment for Darcy-Forchheimer Flow of Sisko Nanomaterial with Nonlinear Thermal Radiation by Lobatto IIIA Technique
}

\author{
Iftikhar Uddin, ${ }^{1,2}$ Rizwan Akhtar ${ }^{(D},{ }^{1}$ Zhu Zhiyu, ${ }^{1}$ Saeed Islam $\left(\mathbb{D},{ }^{2}\right.$ \\ Muhammad Shoaib, ${ }^{3}$ and Muhammad Asif Zahoor Raja ${ }^{4}{ }^{4}$ \\ ${ }^{1}$ School of Electronics and Information, Jiangsu University of Science and Technology, Zhenjiang, China \\ ${ }^{2}$ Department of Mathematics, Abdul Wali Khan University Mardan (KPK), 23200, Pakistan \\ ${ }^{3}$ Department of Mathematics, COMSATS University Islamabad, Attock Campus, Pakistan \\ ${ }^{4}$ Department of Electrical and Computer Engineering, COMSATS University Islamabad, Attock Campus, Pakistan
}

Correspondence should be addressed to Rizwan Akhtar; rizwan@just.edu.cn

Received 17 February 2019; Accepted 17 April 2019; Published 13 May 2019

Academic Editor: Mohamed Abd El Aziz

Copyright (C) 2019 Iftikhar Uddin et al. This is an open access article distributed under the Creative Commons Attribution License, which permits unrestricted use, distribution, and reproduction in any medium, provided the original work is properly cited.

In this study, the competency of numerical computational framework based on Lobatto IIIA technique is utilized for dynamical analysis of the Darcy-Forchheimer flow of Sisko nanomaterial with nonlinear thermal radiation. The resultant PDEs of the Sisko fluid model expressions are transformed into system of nonlinear ODEs by exploiting the similarity variables. Graphical representations and numerical illustrations are used to envisage the characteristics of various physical parameters of interest on velocity profile, nanoparticles concentration, and temperature distribution of Sisko fluidic system. In addition, skin friction and Nusselt number are numerically examined with observation that the material parameter of Sisko fluid increases the velocity profile as well as Nusselt number while decreasing temperature, concentration profiles, and skin friction coefficient.

\section{Introduction}

Material of the type having pores is termed as porous medium, in fluid dynamics; usually it is considered that the porous medium is filled with nanofluid. The study of nanofluid flow through porous medium has wide range of applications due to the fact that it has become a field of high interest for researchers, mathematicians, engineers, geologists, and architectures. Some of the considerable applications are flow of liquid in reservoirs, catalytic reactors, production of oil, heat exchanger, etc. Transfer of heat in porous medium has extensively been used in the heat pipe technology, nonwoven ingredients, production of papers, electronic based technology, storage of energy, etc. [1-4]. Henry Darc, in 1856, a French engineer, pioneered the flow of homogeneous liquids through porous medium during his valuable work on liquid flow through the sand beds. The law of Darcy is not effective for the situation when boundary and inertial effects appears at higher rate of fluid flow. After that the work was extended by Dutch scientist named Philippes Forchheimer in 1901. He extended the velocity equation by adding the square of velocity term in the equation of momentum of the problem to predict the behavior of boundary layer flow and inertia [5]. The term Forchheimer was later introduced by Muskat [6]. Pal and Mondal [7] constructed a fluid model with DarcyForchheimer effects over stretching sheet and he examined that with the decrease of the nanoparticle concentration profile, the value of electric field parameter increased. Ganesh et al. [8] studied the Darcy-Forchheimer MHD nanoliquid.

Thermal radiation, either linear or nonlinear, has a very significant role in different high temperature processes in the field of fluid dynamics. It has diverse uses in several types of power plants and industries. For final possessions of desired qualities, the control of heat transfer is very significant and necessary in industries. Modern system linked to astrophysical flow, reactors cooling, electrical power generators, vehicles in the space, and plasmas are working by useful applications of radiation either linear or nonlinear. 
Latif et al. [9] studied the nonuniform viscosity fluid model with nonlinear radiation. Unsteady flow in existence of source/sink and radiation effects is modeled by Hayat et al. [10]. Pal [11] studied thermal radiation in a channel the peristaltic transport by using convective conditions. Pandey and kumar [12] studied the thermal radiation as well as mass flux in porous medium on natural convection. The effect of thermal radiation natural convection has been investigated by Huang [13]. Kothandapani and Prakash [14] worked out upon the influence of radiation over the peristalsis of magneto hydrodynamics in a channel. Some renowned researchers also worked on thermal radiation [15-23]. In these studies, the radiation with nonlinearity was introduced by the researchers with in fluid flow models. In these investigations extra variable contains difference between temperature of wall and ambient temperature. Due to high temperature variation, the transfer of radiation amongst the two objects is fortified. More details regarding nonlinear radiation are given [24-33]. Some recent work related to Non-Newtonian fluids, radiation, porous medium, and nanofluid is provided here [34-37]. These are the motivating factors for the authors to investigate in the model and analysis of nonlinear radiated nanofluid flow of Sisko fluid. The noticeable features of the study are listed as follows.

(i) An innovative design of Sisko nanofluid with nonlinear radiation and Darcy-Forchheimer flow involving porous medium has been modeled and PDEs of the model are transformed into an equivalent system of ODEs using suitable similarity transformations.

(ii) The concept of boundary layer is incorporated to develop the fluidic problem. The energy expression of the problem is developed by adding nonlinear radiation term.

(iii) Numerical analysis of the Sisko fluid model involving nanoparticles is presented by invoking the Lobatto IIIA method in order to investigate the effects of involved physical variables on velocity, concentration, and temperature by variation of physical quantities.

(iv) The application of Lobatto IIIA method to this model is an innovative work with these effects. Lobatto IIIA is a version of bvp $4 \mathrm{c}$ method, which is based on finite difference method. Other methods can also solve this problem, i.e., NDSolve, RK-4, HAM, etc. this method has a strength to solve highly nonlinear systems of ODEs.

(v) Results presented in numerical and graphical representations endorsed the worth of the methodology as viable and effective computing solver for visualizing the dynamics of Sisko nanofluidic model.

Outline of the rest of the paper is given as follows: mathematical model of the problem is represented in Section 2, in Section 3, results and discussion of numerical experiments are provided, while the conclusion and future research work are presented in the last section.

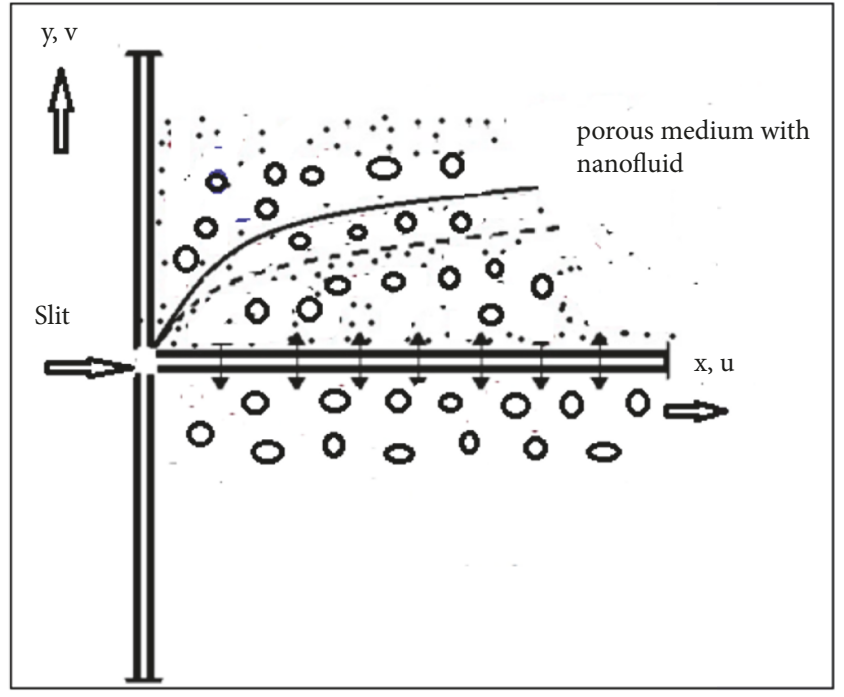

Figure 1: Geometry representing the problem.

\section{Mathematical Development}

Consider two-dimensional Sisko nanofluid flow through a porous medium and nonlinear stretching sheet as shown in Figure 1. The coordinate $y$ is taken as normal to the sheet while $\mathrm{x}$-axis is along the sheet. A hot fluid is taken for heating or cooling the surface of sheet connectively. It is considered that uniformly concentrated fluid with nanoparticles is taken at the surface of the stretching sheet. The two dimensional governing equations with boundary layer are given below $[38,39]$ :

$$
\begin{aligned}
\left(\frac{\partial u}{\partial x}+\frac{\partial v}{\partial y}\right)= & 0, \\
u \frac{\partial u}{\partial x}+v \frac{\partial u}{\partial y}= & \frac{a}{\rho} \frac{\partial^{2} u}{\partial y^{2}}-\frac{b}{\rho} \frac{\partial}{\partial y}\left(-\frac{\partial u}{\partial y}\right)^{n}-\frac{v}{K} u \\
& -F u^{2}, \\
\left(u \frac{\partial T}{\partial x}+v \frac{\partial T}{\partial y}\right)= & \alpha \frac{\partial^{2} T}{\partial y^{2}} \\
& +\tau\left[D_{B} \frac{\partial C}{\partial y} \frac{\partial T}{\partial y}+\frac{D_{T}}{T_{\propto}}\left(\frac{\partial T}{\partial y}\right)^{2}\right] \\
& -\frac{1}{\rho c_{p}} \frac{\partial q_{r}}{\partial y}, \\
\left(u \frac{\partial C}{\partial x}+v \frac{\partial C}{\partial y}\right)= & D_{B}\left(\frac{\partial^{2} C}{\partial y^{2}}\right)+\frac{D_{T}}{T_{\propto}}\left(\frac{\partial^{2} T}{\partial y^{2}}\right),
\end{aligned}
$$

with the boundary conditions

$$
\begin{aligned}
& y=0, \\
& u=u_{w}(x)=c x^{s}, \\
& v=0,
\end{aligned}
$$




$$
\begin{aligned}
-k \frac{\partial T}{\partial y} & =h_{f}\left(T_{f}-T\right), \\
\mathrm{C} & =C_{w}, \\
y & \longrightarrow \infty: u \longrightarrow 0, \\
T & \longrightarrow T_{\infty}, \\
\mathrm{C} & \longrightarrow \mathrm{C}_{\infty},
\end{aligned}
$$

where the inertia coefficient $F$ is defined by

$$
F=\frac{C_{b}}{x \sqrt{K}}
$$

Rosseland radiation approximation is given as

$$
q_{r}=-\frac{4 \sigma^{*}}{3 k^{*}} \frac{\partial T^{4}}{\partial y}
$$

Term $\sigma^{*}$ in the above equation is Stefan-Boltzmann parameter while $k^{*}$ is mean absorption parameter. After simplifying (7), we have

$$
\frac{\partial q_{r}}{\partial y}=-\frac{16 \sigma^{*}}{3 k^{*}} \frac{\partial T_{\infty}^{3}}{\partial y} \frac{\partial^{2} T}{\partial y^{2}}
$$

In order to obtain ODEs from the above PDEs, we use the similarity transformations

$$
\begin{aligned}
& u=c x^{s} f^{\prime} \\
& v=-U R_{e b}^{-1 /(n+1)} \\
& \frac{1}{n+1}\left\{[s(2 n-1)+1] f+[s(2-n)-1] \eta f^{\prime}\right\} \\
& \eta=\frac{y}{x} R_{e b}^{1 /(n+1)} \\
& \psi=x U R_{e b}^{-1 /(n+1)} f(\eta), \\
& \phi(\eta)=\frac{C-C_{\infty}}{C_{w}-C_{\infty}} \\
& \theta(\eta)=\frac{T-T_{\infty}}{T_{f}-T_{\infty}} .
\end{aligned}
$$

Continuity equation is identically satisfied, while (2)-(4) yield

$$
\begin{aligned}
& A f^{\prime \prime \prime}+n\left(-f^{\prime \prime}\right)^{n-1} f^{\prime \prime \prime}+\left(\frac{s(2 n+1)+1}{n+1}\right) f f^{\prime \prime} \\
& -s\left(f^{\prime}\right)^{2}-k_{1} f^{\prime}-\operatorname{Fr}\left(f^{\prime}\right)^{2}=0,
\end{aligned}
$$

$$
\begin{aligned}
\theta^{\prime \prime} & +\operatorname{Pr}\left(\frac{s(2 n+1)+1}{n+1}\right) f \theta^{\prime}+\left(1+\frac{4}{3} R d\right) \theta^{\prime \prime}+\frac{4}{3} \\
& \cdot \operatorname{Rd}\left[\left(\theta_{w}-1\right)^{3}\left(3 \theta^{\prime 2} \theta^{2}+\theta^{3} \theta^{\prime \prime}\right)\right. \\
& +3\left(\theta_{w}-1\right)^{2}\left(2 \theta^{\prime 2} \theta+\theta^{2} \theta^{\prime \prime}\right) \\
& \left.+3\left(\theta_{w}-1\right)\left(\theta^{\prime 2}+\theta \theta^{\prime \prime}\right)\right]+\left(\operatorname{Pr} \lambda \theta+N b \theta^{\prime} \phi^{\prime}\right. \\
& \left.+N t\left(\theta^{\prime}\right)^{2}\right)=0
\end{aligned}
$$$$
\phi^{\prime \prime}+\operatorname{Pr} L e\left(\frac{s(2 n-1)+1}{n+1}\right) f \phi+\frac{N t}{N b} \theta^{\prime \prime}=0,
$$

with boundary conditions

$$
f(\eta)=0 \text {, }
$$

$$
\begin{aligned}
& f^{\prime}(\eta)=1, \\
& \theta^{\prime}(\eta)=-\gamma(1-\theta(\eta)), \\
& \phi(\eta)=1
\end{aligned}
$$

at $\eta=0$,

$$
\begin{gathered}
f^{\prime}(\eta) \longrightarrow 0 \\
\theta(\eta) \longrightarrow 0 \\
\phi(\eta) \longrightarrow 0
\end{gathered}
$$

as $\eta \longrightarrow \infty$,

where parameters appearing in (9)-(16) are defined as

$$
\begin{aligned}
\operatorname{Re}_{a} & =\rho \frac{\mathrm{U}_{x}}{a}, \\
\operatorname{Re}_{b} & =\frac{\rho x^{n} U^{2-n}}{b}, \\
A & =\frac{\operatorname{Re}_{b}^{2 /(n+1)}}{\operatorname{Re}_{a}}, \\
k_{1} & =\frac{a v \mathrm{Re}_{a}}{K \rho U^{2}}, \\
\lambda & =\frac{a \mathrm{Q}_{0} \operatorname{Re}_{a}}{c_{p} \rho U^{2}}, \\
L e & =\frac{\alpha}{D_{B}}, \\
\operatorname{Pr} & =\left(\frac{a \operatorname{Re}_{a}}{\alpha \rho}\right) \operatorname{Re}_{b}^{-2 /(n+1)}, \\
\gamma & =\frac{h}{k} \sqrt{\frac{v}{c}},
\end{aligned}
$$




$$
\begin{aligned}
N b & =\frac{\tau D_{B}}{\alpha}\left(C_{w}-C_{\infty}\right), \\
F r & =\frac{C_{b}}{K^{1 / 2}}, \\
N t & =\frac{D_{T}}{T_{\infty}} \frac{\tau}{\alpha}\left(T_{f}-T_{\infty}\right), \\
\alpha & =\frac{k}{\left(\rho c_{p}\right)_{f}} \\
\tau & =\frac{\left(\rho C_{p}\right)_{s}}{\left(\rho C_{p}\right)_{f}} \\
R d & =\frac{4 \sigma T_{\infty}^{3}}{k^{*} k_{\infty}}
\end{aligned}
$$

where $A$ is material parameter of Sisko fluid. The local Nusselt number is

$$
\begin{aligned}
N u_{x} & =\frac{x q_{w}}{k\left(T_{f}-T_{\infty}\right)}, \\
N u_{x} \mathrm{Re}_{b}^{-1 /(n+1)} & =-\theta^{\prime}(0),
\end{aligned}
$$

and $q_{w}$ is the heat flux, given by

$$
q_{w}=-k\left(\frac{\partial T}{\partial y}\right)_{y=0}
$$

\section{Results and Discussion}

The transformed fluid flow problem defined in (10)-(13) has been solved numerically by exploiting the strength of Labotto IIIA method and invoking MATLAB. routine "bvp4c" for solution of boundary value problem of differential equation. We refer the interested reader to the article [40] for the necessary additional details of the Labotto III solver in [40] and citations therein. The solutions of (10)-(13) are also calculated with explicit Runge-Kutta (RK) method by using the Mathematica routine "NDSolve" for numerical solution of differential equations. The results of Lobatto III A are compared with explicit RK method and error analysis shows the matching of the order $10^{-08}$ to $10^{-09}$ exist between both methodologies which validate the correctness of the proposed numerical procedure. Therefore, in the rest of the study numerical procedure of Labotto IIIA is used for numerical solution of the problem.

Characteristics of some influential parameters like heat Prandtl number Pr, the Froude number Fr, Brownian motion $\mathrm{Nb}$ parameter, thermophoresis number $\mathrm{Nt}$, Lewis number Le, fitted rate constant $n$, the material parameter of Sisko fluid $\mathrm{A}$, the radiation parameter $\mathrm{Rd}$, the porosity permeability parameter $k_{1}$, the heat generation/absorption variable $\lambda$, the Biot number $\gamma$, and $\theta_{w}$ the temperature ratio parameter on the velocity $f^{\prime}(\eta)$, temperature $\theta(\eta)$, and concentration $\phi(\eta)$ are shown. These numerical outcomes have been illustrated in Figures 2-10 and Tables 1-7. Numerical computation has been performed by choosing the default estimations of involved variables in the problem as $\mathrm{Fr}=n=A=\lambda=0.5$, $\operatorname{Pr}=1.2, L e=1.5, N b=N t=0.2, s=1.5, k 1=0.1, \gamma=1$, $R d=0.9$, and $\theta_{w}=0.7$. Figures 2(a), 2(b), and 2(c) show the influence of Fr on $f(\eta), f^{\prime}(\eta)$, and $\phi(\eta)$. For increasing values of $\mathrm{Fr}, f(\eta)$ and $f^{\prime}(\eta)$ decrease while $\phi(\eta)$ increases. Figure 2(d) discloses the effect of $n$ on $f(\eta)$. By increasing $\mathrm{n}, f(\eta)$ shows decreasing behavior. Figures 3(a), 3(b), and $3(\mathrm{c})$ depict the decreasing behavior of $f^{\prime}(\eta), \theta(\eta)$, and $\phi(\eta)$, respectively, for increasing values of $\mathrm{n}$. In Figure 3(d) increasing quality is illustrated for $\theta(\eta)$ for higher values of Prandtl number Pr. The concentration profile $\phi(\eta)$ shows decreasing characteristic for increasing Prandtl number Pr, Lewis number Le, and Brownian number $\mathrm{Nb}$, which are shown in Figures 4(a), 4(b), and 4(d) respectively. Increase in Lewis Number Le has decreasing effect because the larger Lewis number makes diffusivity of molecules smaller and due to this reason, the concentration field is decreased. The temperature profile $\theta(\eta)$ is enhanced in Figure 4(c) for higher $\mathrm{Nb}$. In Figures 5(a) and 5(b), it is clearly seen that increasing Nt causes increasing temperature $\theta(\eta)$ and concentration $\phi(\eta)$ profiles. Enhancement in temperature profile due to increase in thermophoresis number is because of nanoparticles. The nanoparticles which are closer to hot fluid shift towards cold fluid due to increase in thermophoresis parameter, which makes the temperature boundary layer thick. It is obvious in Figures 5(c) and 5(d) that increase in A causes increase in $f(\eta)$ and $f^{\prime}(\eta)$, respectively. From Figures 6(a) and 6(b), it is clear that temperature $\theta(\eta)$ and concentration $\phi(\eta)$, respectively, both are decreasing for higher values of A. Also, Figures 6(c) and 6(d) both represent the decreasing behavior of $f(\eta)$ and $f^{\prime}(\eta)$, respectively, for enhanced values of parameter s. Enhancement is seen in $\theta(\eta)$ and $\phi(\eta)$ for increase in s, which is shown in Figures 7(a) and 7(b), respectively. An increase in $k_{1}$ results in decrease in $f(\eta)$ and $f^{\prime}(\eta)$, which is visualized in Figures $7(\mathrm{c})$ and $7(\mathrm{~d})$, respectively, and increasing effect of $k_{1}$ is shown for $\theta(\eta)$ and $\phi(\eta)$ for increasing values of $k_{1}$ in Figures 8(a) and 8(b), respectively. It is found in Figures $8(\mathrm{c})$ and $8(\mathrm{~d})$ that $\theta(\eta)$ and $\phi(\eta)$ are enhanced, respectively, for higher estimations of $\gamma$. Figures 9(a), 9(b), 9(c), and $9(\mathrm{~d})$ visualize the effect of $\lambda$ on $f(\eta), f^{\prime}(\eta), \theta(\eta)$, and $\phi(\eta)$, respectively. Higher estimations of $\lambda$ cause decrease in $f(\eta)$ and $f^{\prime}(\eta)$, while increase in $\theta(\eta)$ and dual behavior in concentration profile $\phi(\eta)$. In the domain from 0 to $2, \phi(\eta)$ decreases while in the domain from 2 to $7 \phi(\eta)$ it shows opposite behavior. Figure 10(a) shows that increase in $\mathrm{Rd}$ causes decrease in $\theta(\eta)$ and dual behavior of $\phi(\eta)$ is seen for higher estimations of Rd in Figure 10(b). In the domain from 0 to $2, \phi(\eta)$ increases while in the domain from 2 to 7 , $\phi(\eta)$ decreases. Figure 10(c) discloses the increasing behavior of $\theta(\eta)$ for higher values of the temperature ratio parameter $\theta_{w}$ on temperature distribution $\theta(\eta)$. This is because greater ratio in the temperature increases the temperature boundary layer. Figure 10(d) implies the dual characteristic of $\phi(\eta)$ for higher values of $\theta_{w}$. In the domain from 0 to 2 , $\phi(\eta)$ diminishes while in domain from 2 to $7, \phi(\eta)$ enhances. Table 1 shows the variation of values of involved parameters in different scenarios. Scenario 1 shows the variation of Fr; 


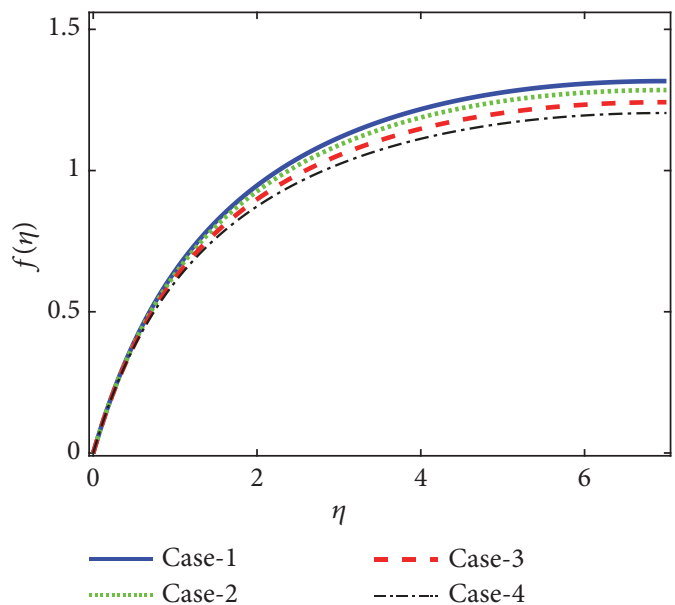

(a) Effect on $f(\eta)$ by varying $\mathrm{Fr}$

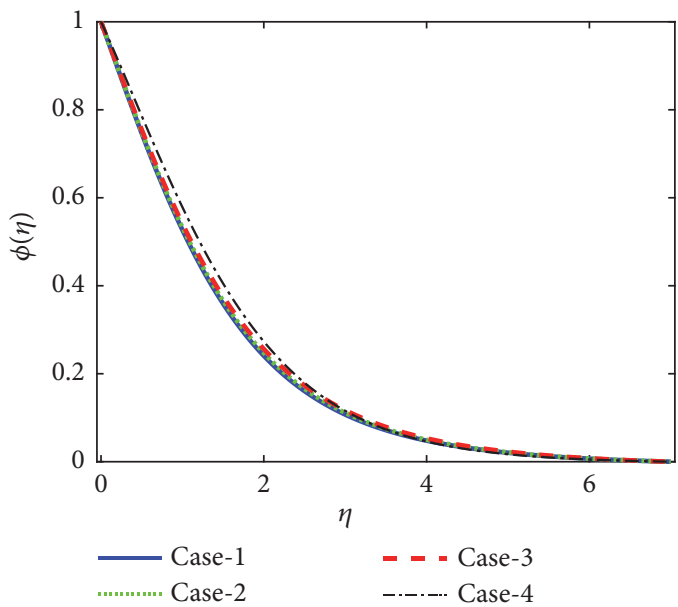

(c) Effect on $\phi(\eta)$ by varying Fr

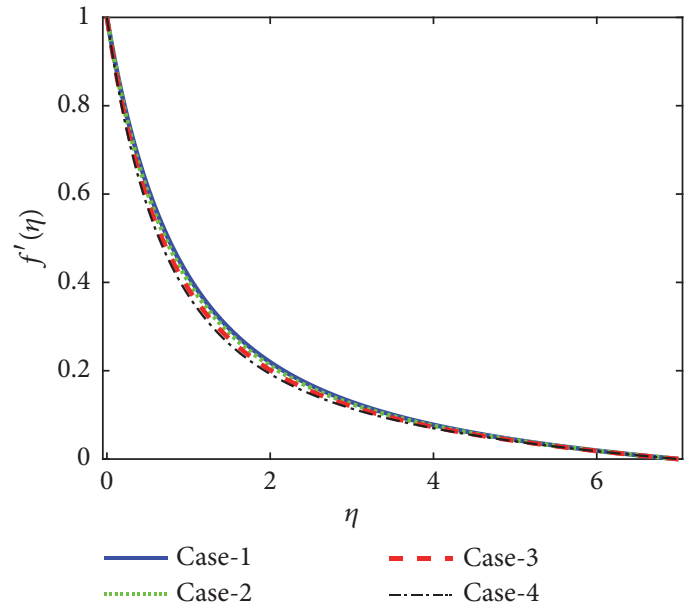

(b) Effect on $f^{\prime}(\eta)$ by varying $\mathrm{Fr}$

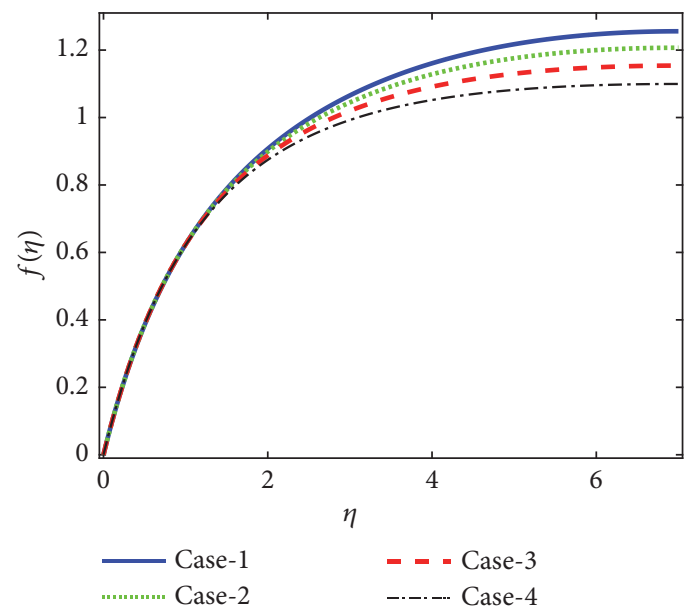

(d) Effect on $f(\eta)$ by varying $\mathrm{n}$

Figure 2: Variation of Fr on $f, f^{\prime}$ and $\phi$, while n on $f$.

TABle 1: Parameters of physical quantities in the Sisko nano fluid model.

\begin{tabular}{|c|c|c|c|c|}
\hline SCENARIOS & Case-1 & Case-2 & Case- 3 & Case- 4 \\
\hline 1 & $\mathrm{Fr}=0.1$ & $\mathrm{Fr}=0.3$ & $\mathrm{Fr}=0.6$ & $\mathrm{Fr}=0.9$ \\
\hline 2 & $\mathrm{n}=0.5$ & $\mathrm{n}=0.6$ & $\mathrm{n}=0.7$ & $\mathrm{n}=0.8$ \\
\hline 3 & $\operatorname{Pr}=1.0$ & $\operatorname{Pr}=1.2$ & $\operatorname{Pr}=1.4$ & $\operatorname{Pr}=1.6$ \\
\hline 4 & $\mathrm{Le}=1.2$ & $\mathrm{Le}=1.4$ & $\mathrm{Le}=1.6$ & $\mathrm{Le}=1.8$ \\
\hline 5 & $\mathrm{Nb}=0.7$ & $\mathrm{Nb}=0.9$ & $\mathrm{Nb}=1.3$ & $\mathrm{Nb}=1.8$ \\
\hline 6 & $\mathrm{Nt}=0.2$ & $\mathrm{Nt}=0.4$ & $\mathrm{Nt}=0.6$ & $\mathrm{Nt}=0.8$ \\
\hline 7 & $A=0.2$ & $\mathrm{~A}=0.4$ & $A=0.6$ & $A=0.8$ \\
\hline 8 & $\mathrm{~s}=1.1$ & $\mathrm{~s}=1.3$ & $\mathrm{~s}=1.5$ & $\mathrm{~s}=1.7$ \\
\hline 9 & $k 1=0.1$ & $k 1=0.3$ & $k 1=0.5$ & $k 1=0.7$ \\
\hline 10 & $\gamma=1.0$ & $\gamma=1.2$ & $\gamma=1.4$ & $\gamma=1.6$ \\
\hline 11 & $\lambda=0.2$ & $\lambda=0.4$ & $\lambda=0.6$ & $\lambda=0.8$ \\
\hline 12 & $R d=0.9$ & $R d=1.2$ & $R d=1.5$ & $R d=1.8$ \\
\hline 13 & $\theta_{w}=0.5$ & $\theta_{w}=0.6$ & $\theta_{w}=0.7$ & $\theta_{w}=0.8$ \\
\hline
\end{tabular}




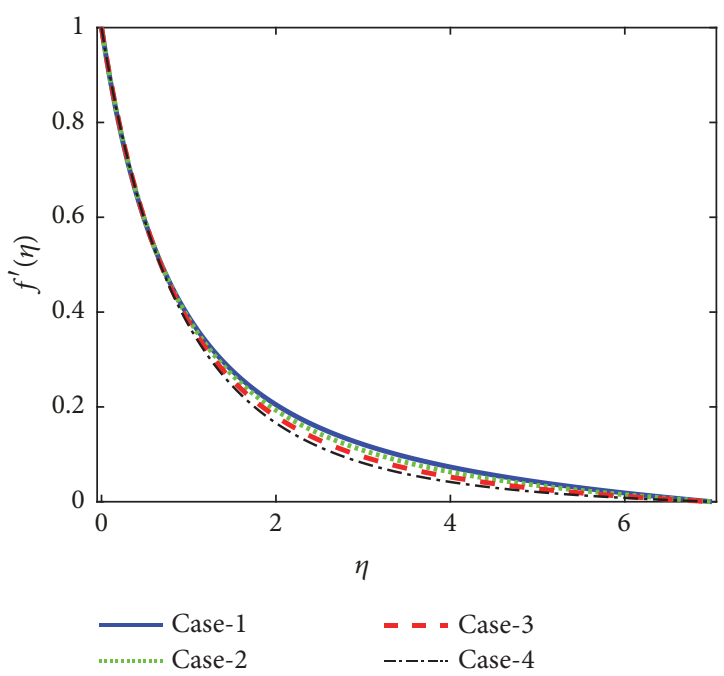

(a) Effect on $f^{\prime}(\eta)$ by varying $\mathrm{n}$

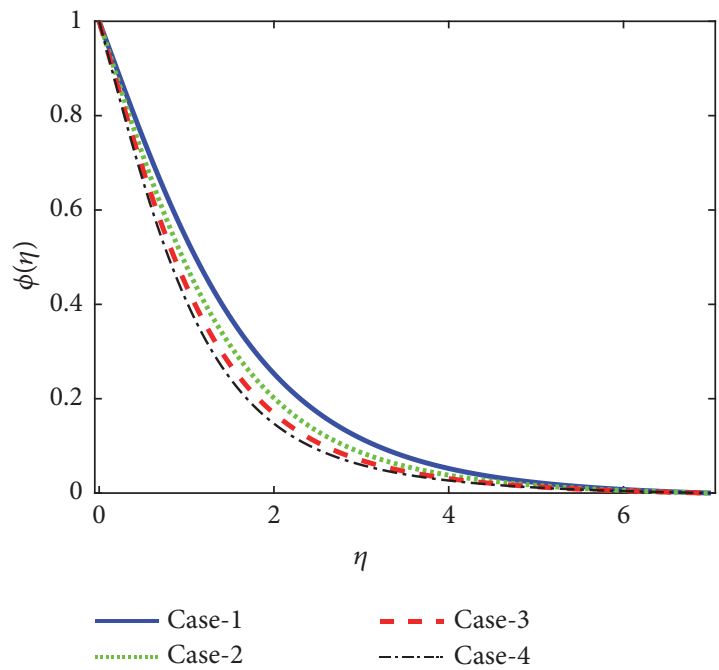

(c) Effect on $\phi(\eta)$ by varying $n$

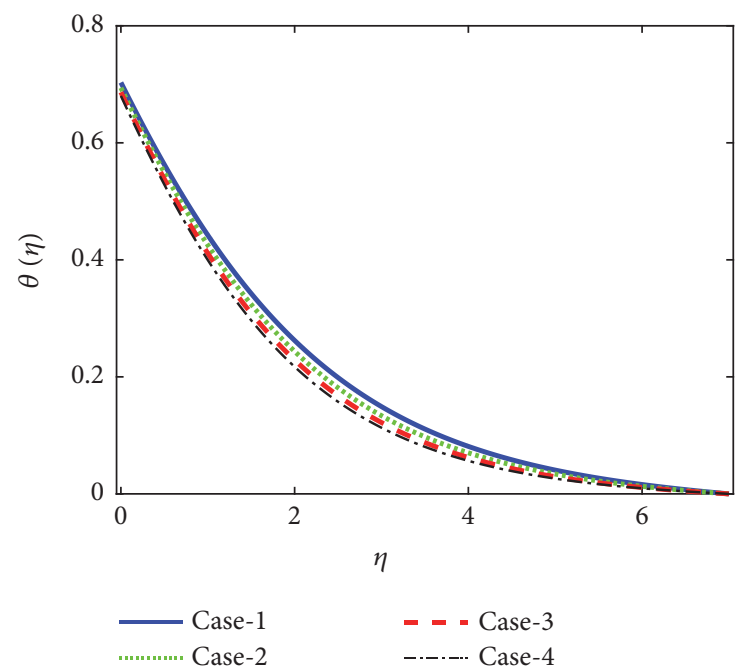

(b) Effect on $\theta(\eta)$ by varying $n$

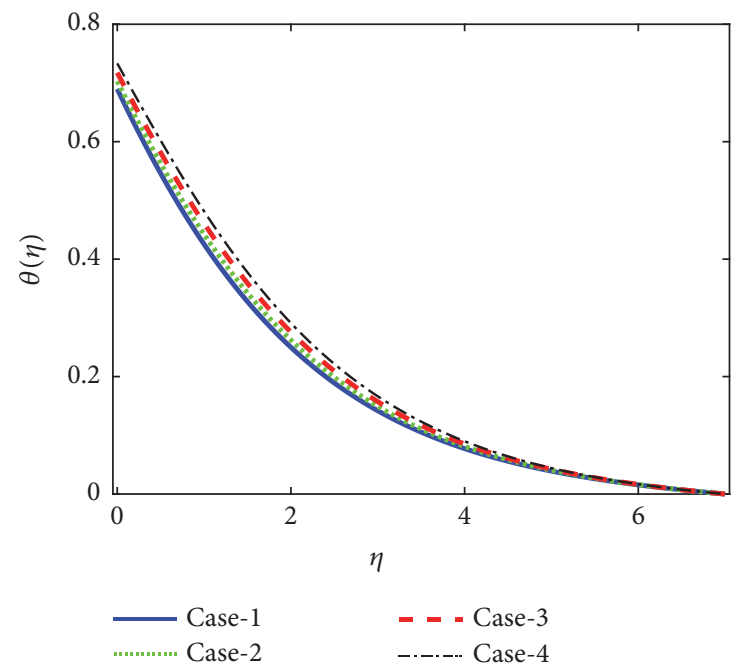

(d) Effect on $\theta(\eta)$ by varying $\operatorname{Pr}$

Figure 3: Variation of $\mathrm{n}$ on $f^{\prime}, \theta$ and $\phi$, while $\operatorname{Pr}$ on $\theta$.

TABLE 2: Numerical data of skin friction $-f^{\prime \prime}(0)$ for the Sisko model.

\begin{tabular}{lllll}
\hline SCENARIOS & Case-1 & Case- 2 & Case-3 & Case-4 \\
\hline 1 & 1.0902 & 1.1504 & 1.2367 & 1.1501 \\
2 & 1.2085 & 1.17529 & 1.2085 & 1.1310 \\
3 & 1.2085 & 1.2085 & 1.2085 & 1.2085 \\
4 & 1.2085 & 1.2085 & 1.2085 & 1.2085 \\
5 & 1.2085 & 1.2085 & 1.2085 & 1.2085 \\
6 & 1.2085 & 1.2085 & 1.1508 & 1.0579 \\
7 & 1.4636 & 1.2768 & 1.2085 & 1.2646 \\
8 & 1.0902 & 1.1504 & 1.3747 & 1.4522 \\
9 & 1.2085 & 1.2936 & 1.2085 & 1.2085 \\
10 & 1.2085 & 1.2085 & 1.2085 & 1.2646 \\
11 & 1.0902 & 1.1504 & 1.2085 & 1.2085 \\
13 & 1.2085 & 1.2085 & 1.2085 & 1.2085 \\
\hline
\end{tabular}




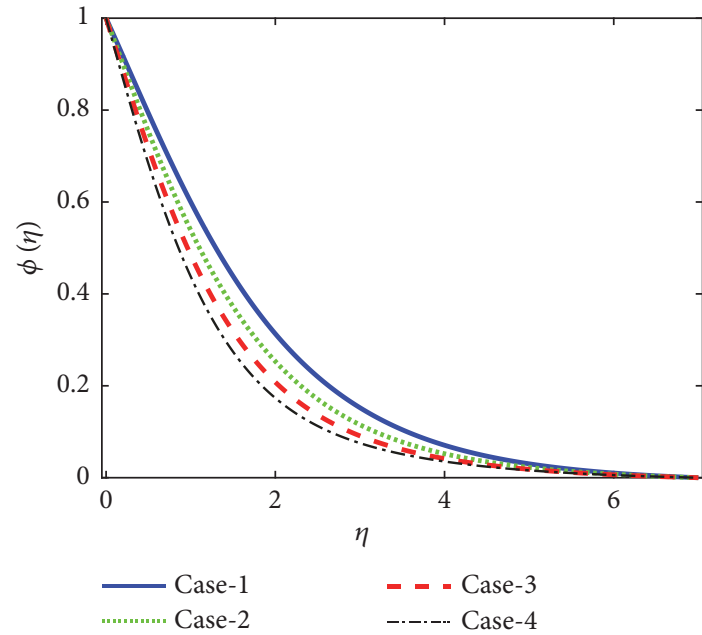

(a) Effect on $\phi(\eta)$ by varying $\operatorname{Pr}$

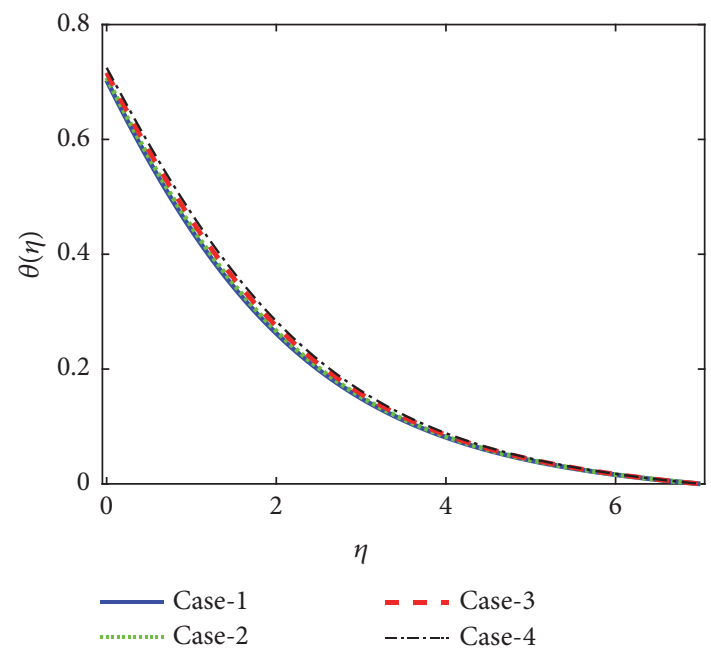

(c) Effect on $\theta(\eta)$ by varying $\mathrm{Nb}$

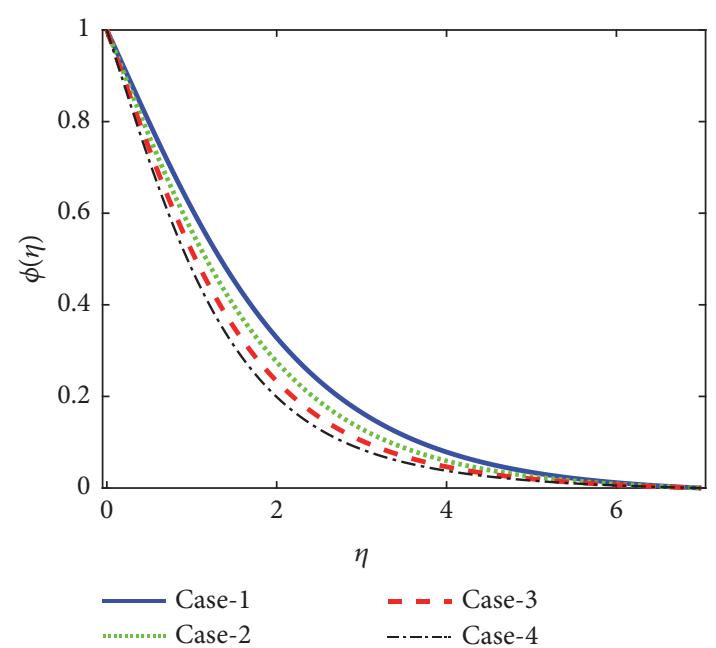

(b) Effect on $\phi(\eta)$ by varying Le

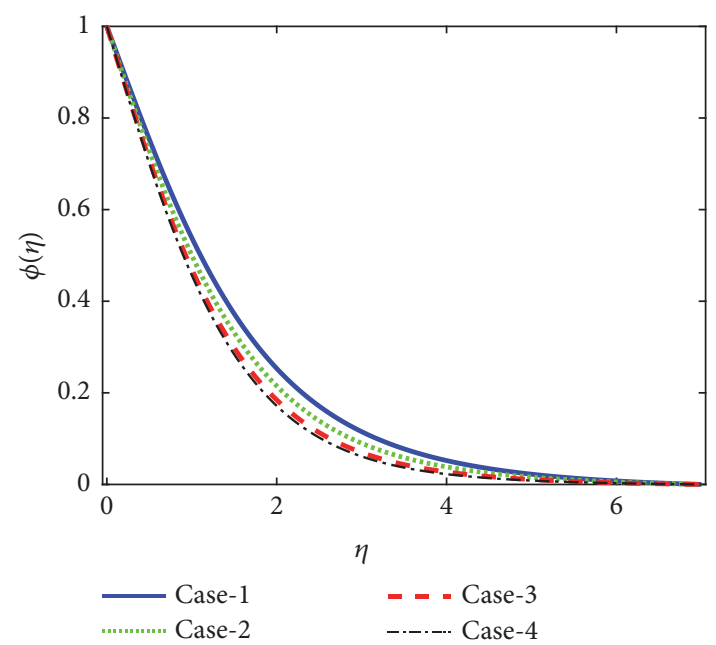

(d) Effect on $\phi(\eta)$ by varying $\mathrm{Nb}$

Figure 4: Variation of $\operatorname{Pr}$ on $\phi$, Le on $\phi, N b$ on $\theta$, and $N b$ on $\phi$.

TABLE 3: Computations for surface heat transfer rate $\left(-\theta^{\prime}(0)\right)$ where: $F r=n=A=\lambda=0.5, \operatorname{Pr}=1.2, L e=1.5, N b=N t=0.2, s=1.5$, $k 1=0.1, \gamma=1, R d=0.9$ and $\theta_{w}=0.7$.

\begin{tabular}{lllll}
\hline SCENARIOS & Case-1 & Case-2 & Case-3 & Case-4 \\
\hline 1 & 0.2984 & 0.2976 & 0.2965 & 0.3812 \\
2 & 0.2968 & 0.3059 & 0.3134 & 0.2824 \\
3 & 0.3100 & 0.2968 & 0.2966 & 0.2666 \\
4 & 0.2976 & 0.2971 & 0.2837 & 0.2961 \\
5 & 0.2968 & 0.2924 & 0.2906 & 0.2751 \\
6 & 0.2968 & 0.2937 & 0.2976 & 0.2875 \\
7 & 0.2940 & 0.2960 & 0.2968 & 0.2961 \\
8 & 0.2984 & 0.2976 & 0.2934 & 0.2920 \\
9 & 0.2968 & 0.2950 & 0.3221 & 0.3308 \\
10 & 0.2968 & 0.3111 & 0.2687 & 0.1791 \\
11 & 0.3571 & 0.3204 & 0.3672 & 0.3868 \\
12 & 0.2968 & 0.3396 & 0.2968 & 0.2174 \\
\hline
\end{tabular}




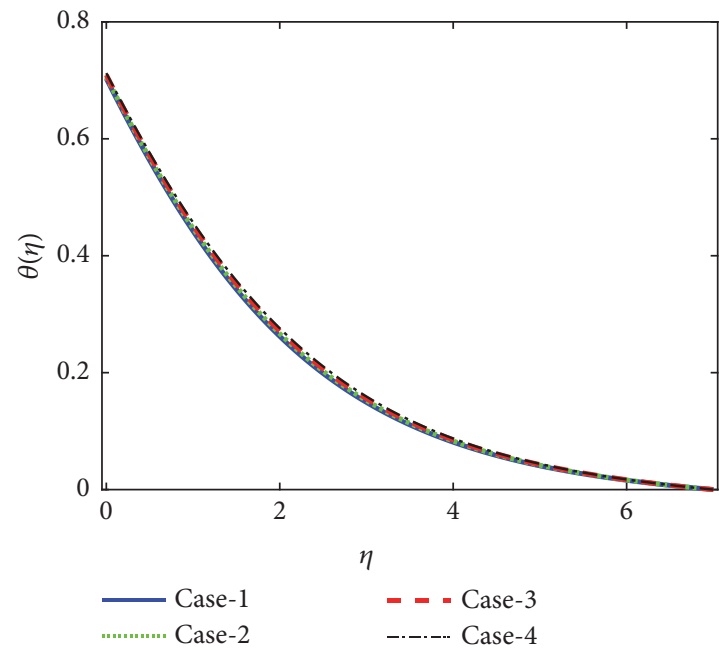

(a) Effect on $\theta(\eta)$ by varying $\mathrm{Nt}$

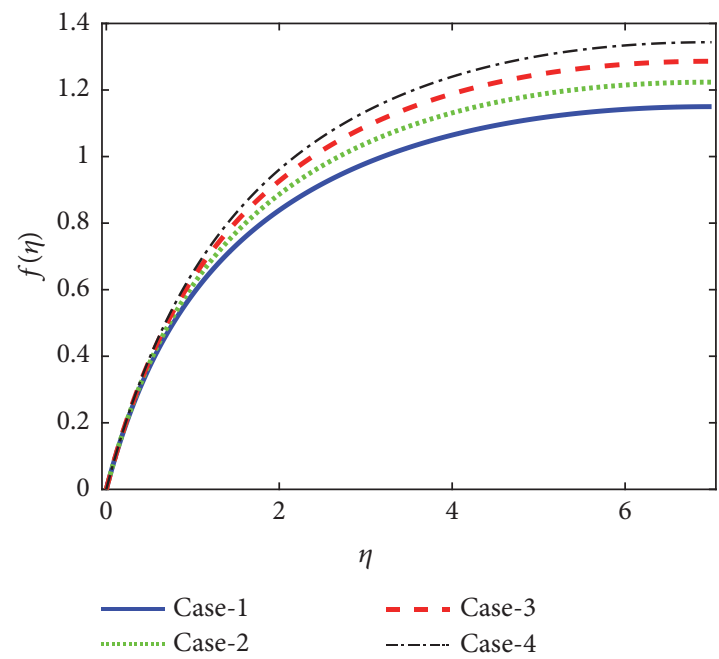

(c) Effect on $f(\eta)$ by varying $\mathrm{A}$

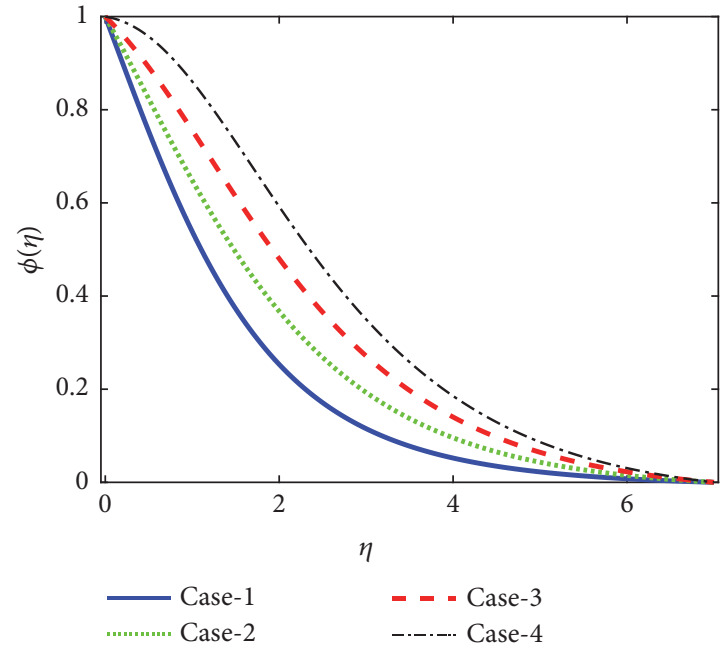

(b) Effect on $\phi(\eta)$ by varying $\mathrm{Nt}$

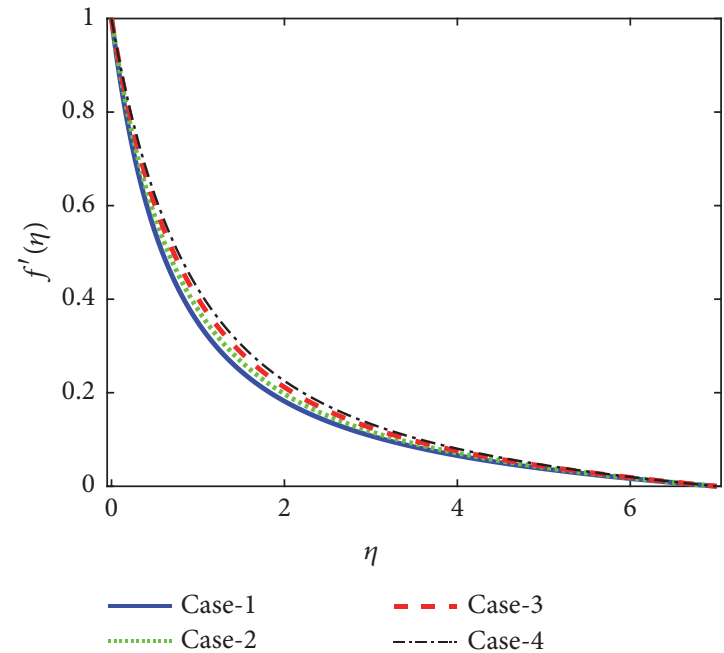

(d) Effect on $f^{\prime}(\eta)$ by varying A

Figure 5: Variation of $N t$ on $\theta$ and $\phi$, while that of $A$ on $f$ and $f^{\prime}$.

TABLE 4: Analysis on the basis of number of meshes of the solver for Sisko model.

\begin{tabular}{lcccc}
\hline SCENARIOS & Case-1 & Case-2 & Case-3 & Case-4 \\
\hline 1 & 866 & 889 & 911 & 1092 \\
2 & 906 & 935 & 906 & 1189 \\
3 & 906 & 906 & 906 & 910 \\
4 & 905 & 906 & 907 & 906 \\
5 & 906 & 906 & 909 & 911 \\
6 & 906 & 914 & 896 & 969 \\
7 & 774 & 924 & 906 & 943 \\
8 & 866 & 889 & 1115 & 1223 \\
9 & 906 & 1012 & 906 & 906 \\
10 & 906 & 906 & 906 & 906 \\
11 & 866 & 889 & 906 & 906 \\
13 & 906 & 906 & 906 \\
\hline
\end{tabular}




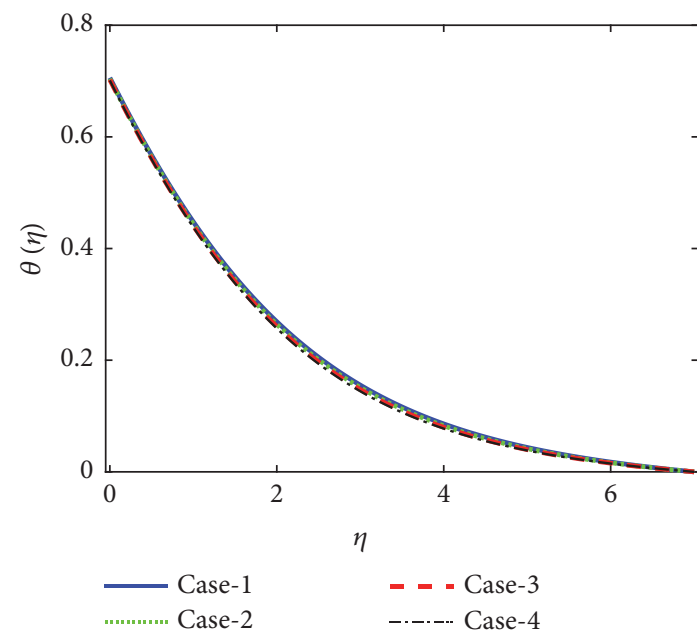

(a) Effect on $\theta(\eta)$ by varying A

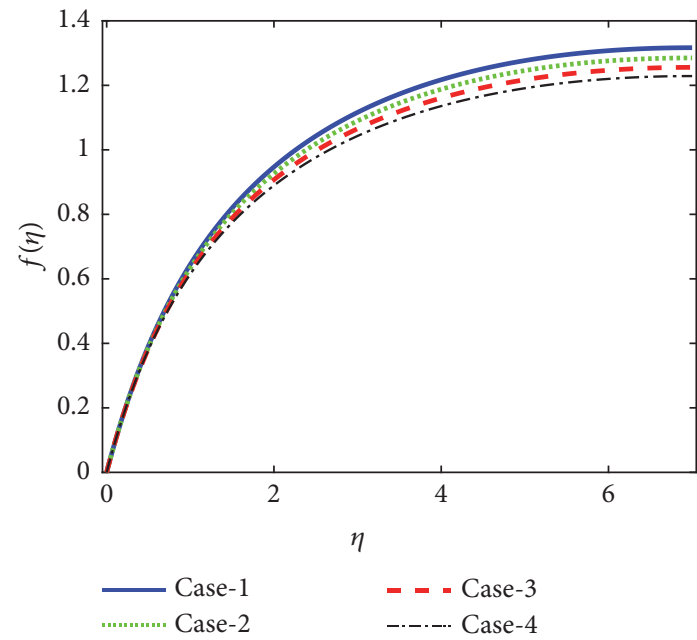

(c) Effect on $f(\eta)$ by varying $s$

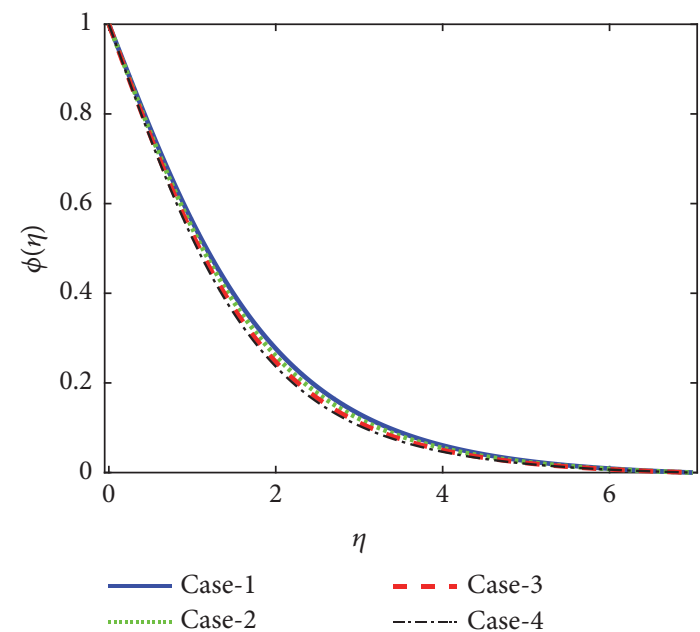

(b) Effect on $\phi(\eta)$ by varying $\mathrm{A}$

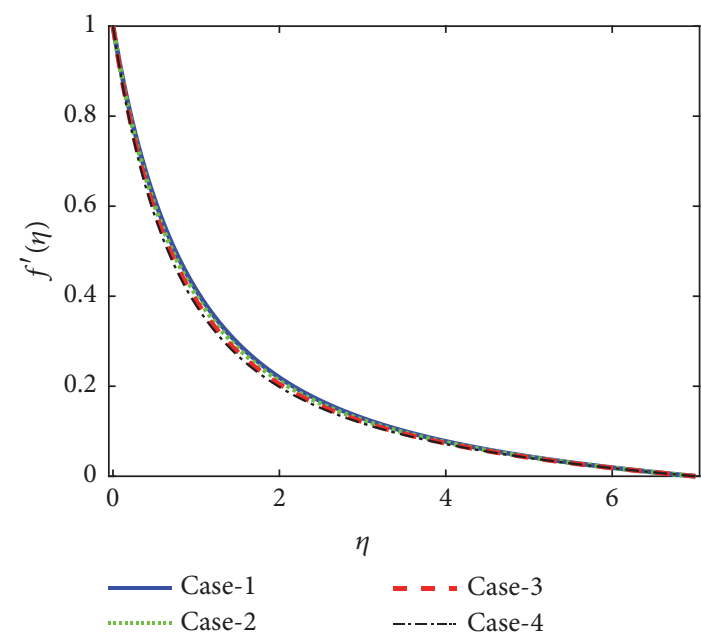

(d) Effect on $f^{\prime}(\eta)$ by varying $s$

FIgURE 6: Variation of A on $\theta(\eta)$ and $\varphi(\eta)$, while that of s on $f(\eta)$ and $f^{\prime}(\eta)$.

TABLE 5: Analysis on the basis of magnitude of relative errors of the solver for Sisko model.

\begin{tabular}{lcccc}
\hline SCENARIOS & Case-1 & Case-2 & Case-3 & Case-4 \\
\hline 1 & $1.451 \mathrm{e}-06$ & $2.990 \mathrm{e}-07$ & $2.487 \mathrm{e}-07$ & $2.247 \mathrm{e}-07$ \\
2 & $2.658 \mathrm{e}-07$ & $4.473 \mathrm{e}-07$ & $4.152 \mathrm{e}-07$ & $7.453 \mathrm{e}-07$ \\
3 & $1.341 \mathrm{e}-07$ & $2.658 \mathrm{e}-07$ & $4.897 \mathrm{e}-07$ & $2.028 \mathrm{e}-07$ \\
4 & $1.228 \mathrm{e}-07$ & $2.017 \mathrm{e}-07$ & $3.300 \mathrm{e}-07$ & $5.433 \mathrm{e}-07$ \\
5 & $2.658 \mathrm{e}-07$ & $3.305 \mathrm{e}-07$ & $2.358 \mathrm{e}-07$ & $1.002 \mathrm{e}-07$ \\
6 & $2.658 \mathrm{e}-07$ & $1.228 \mathrm{e}-07$ & $1.206 \mathrm{e}-07$ & $1.728 \mathrm{e}-07$ \\
7 & $2.768 \mathrm{e}-07$ & $2.266 \mathrm{e}-07$ & $2.256 \mathrm{e}-06$ & $6.363 \mathrm{e}-07$ \\
8 & $1.451 \mathrm{e}-06$ & $2.990 \mathrm{e}-07$ & $2.658 \mathrm{e}-07$ & $2.325 \mathrm{e}-07$ \\
9 & $2.658 \mathrm{e}-07$ & $2.110 \mathrm{e}-07$ & $1.789 \mathrm{e}-07$ & $2.631 \mathrm{e}-07$ \\
10 & $2.658 \mathrm{e}-07$ & $2.464 \mathrm{e}-07$ & $2.343 \mathrm{e}-07$ & $2.304 \mathrm{e}-07$ \\
11 & $1.501 \mathrm{e}-06$ & $2.761 \mathrm{e}-07$ & $2.731 \mathrm{e}-07$ & $1.425 \mathrm{e}-07$ \\
12 & $2.658 \mathrm{e}-07$ & $2.469 \mathrm{e}-07$ & $2.386 \mathrm{e}-07$ & $2.304 \mathrm{e}-07$ \\
13 & $2.604 \mathrm{e}-07$ & $2.431 \mathrm{e}-07$ & $2.658 \mathrm{e}-07$ & $9.887 \mathrm{e}-08$ \\
\hline
\end{tabular}




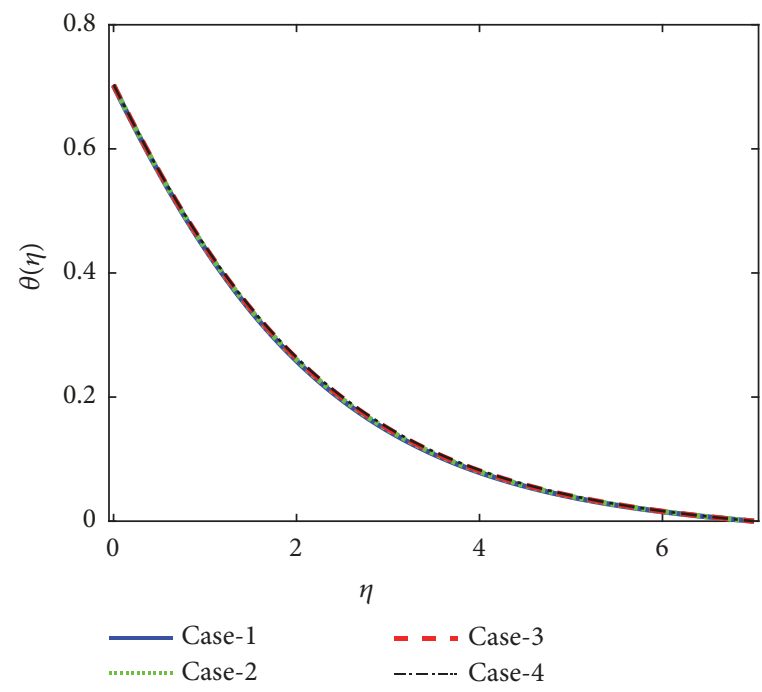

(a) Effect on $\theta(\eta)$ by varying $\mathrm{s}$

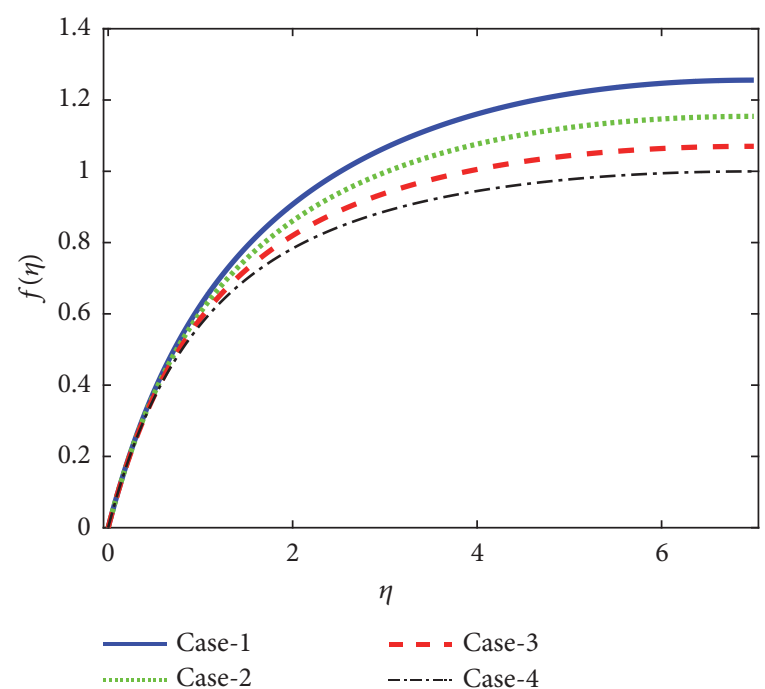

(c) Effect on $f(\eta)$ by varying $k_{1}$

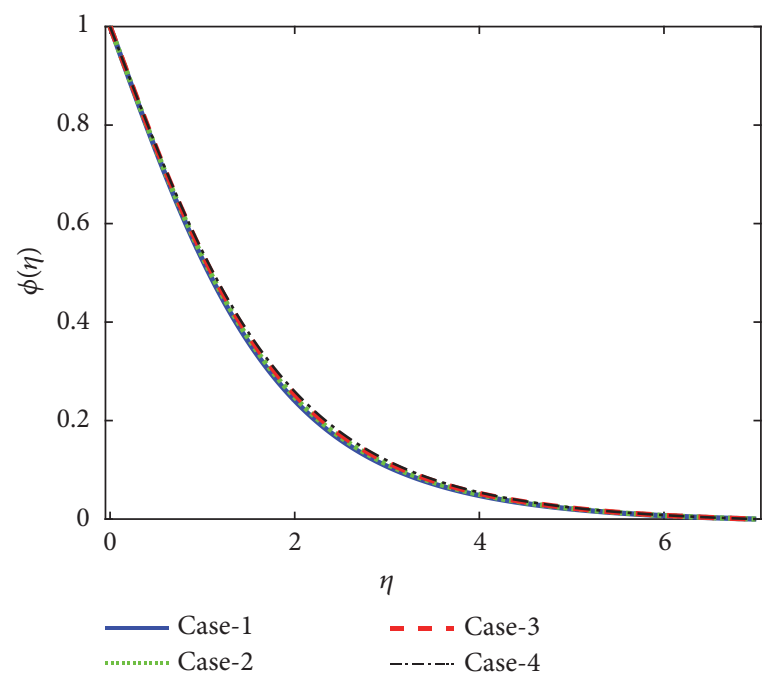

(b) Effect on $\phi(\eta)$ by varying $s$

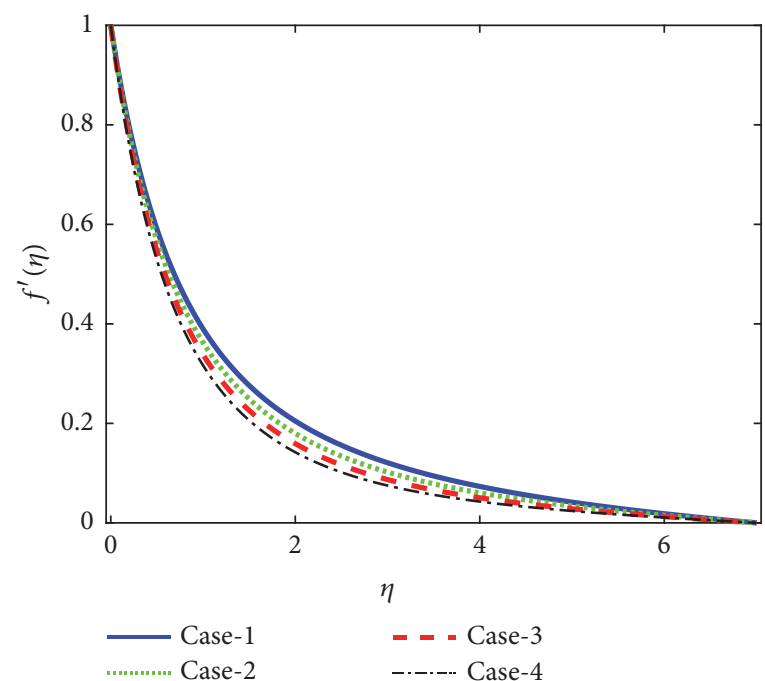

(d) Effect on $f^{\prime}(\eta)$ by varying $k_{1}$

Figure 7: Variation of s on $\theta(\eta)$ and $\varphi(\eta)$, while that of $k_{1}$ on $f(\eta)$ and $f^{\prime}(\eta)$.

TABLE 6: Analysis on the basis of number of ODEs evaluated of the solver for Sisko model.

\begin{tabular}{|c|c|c|c|c|}
\hline SCENARIOS & Case-1 & Case-2 & Case-3 & Case- 4 \\
\hline 1 & 32260 & 34336 & 34666 & 36045 \\
\hline 2 & 34591 & 35025 & 37379 & 38835 \\
\hline 3 & 34591 & 34591 & 34590 & 34649 \\
\hline 4 & 34576 & 34591 & 34590 & 34590 \\
\hline 5 & 34591 & 34591 & 34606 & 34665 \\
\hline 6 & 34591 & 34711 & 34635 & 35535 \\
\hline 7 & 35267 & 34861 & 32650 & 32273 \\
\hline 8 & 32260 & 34336 & 34591 & 35146 \\
\hline 9 & 34591 & 36181 & 37726 & 39346 \\
\hline 10 & 34591 & 34590 & 34590 & 34590 \\
\hline 11 & 32260 & 34337 & 34591 & 35970 \\
\hline 12 & 34591 & 34591 & 34591 & 34591 \\
\hline 13 & 34590 & 34591 & 34591 & 34636 \\
\hline
\end{tabular}




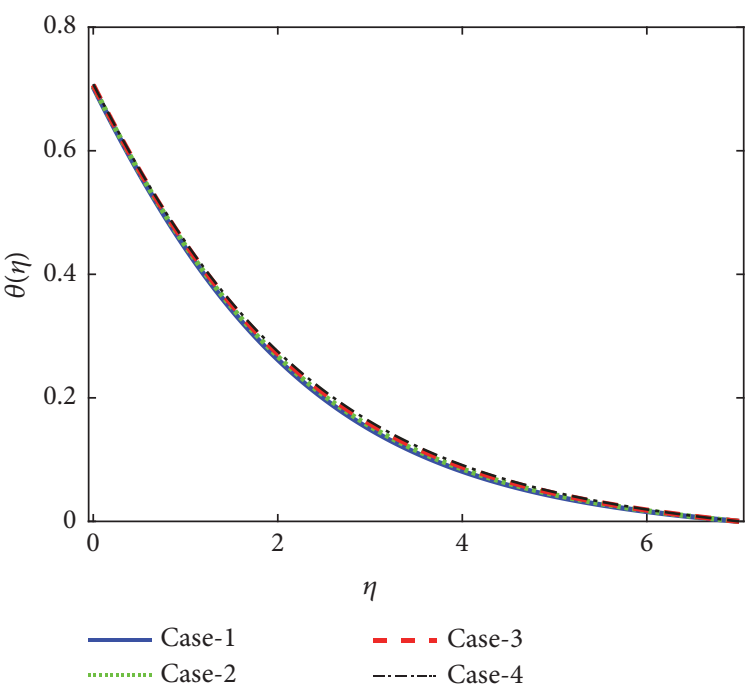

(a) Effect on $\theta(\eta)$ by varying $k_{1}$

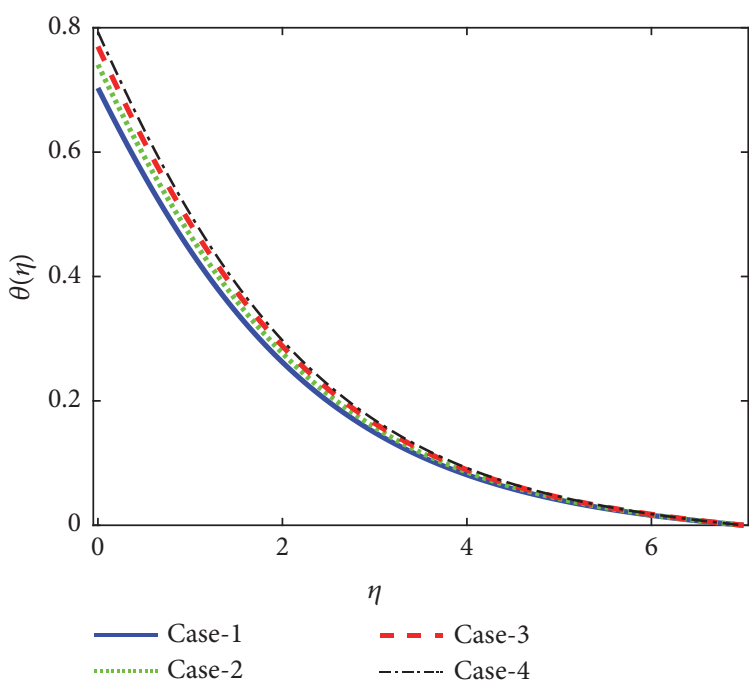

(c) Effect on $\theta(\eta)$ by varying $\gamma$

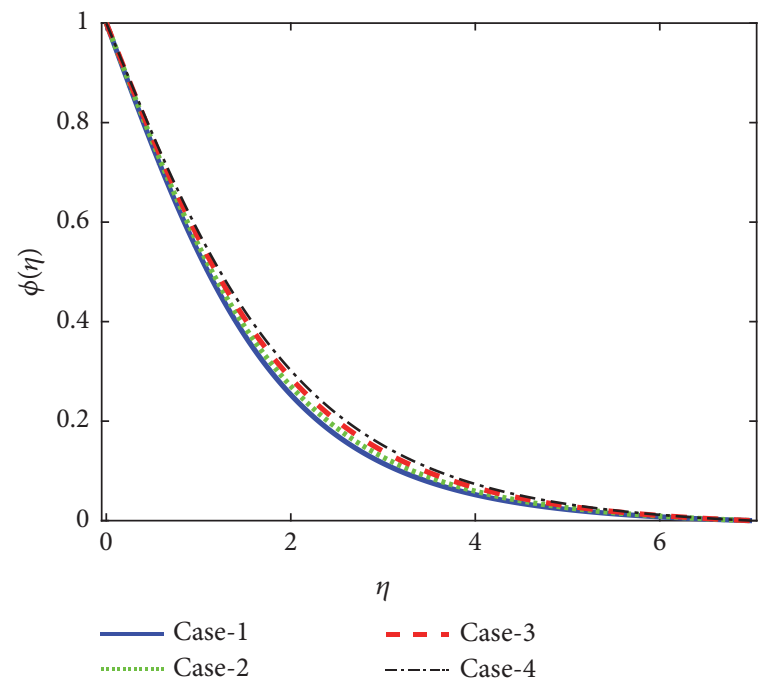

(b) Effect on $\phi(\eta)$ by varying $k_{1}$

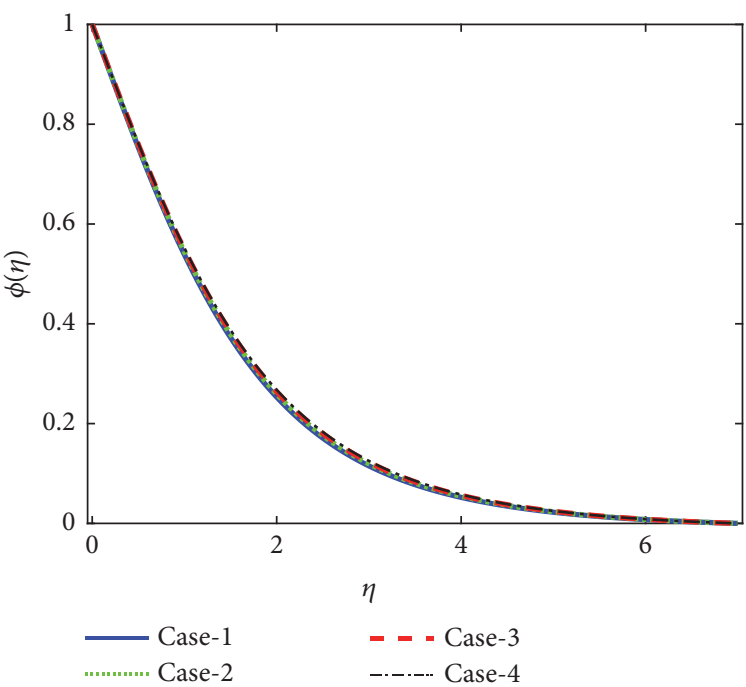

(d) Effect on $\phi(\eta)$ by varying $\gamma$

Figure 8: Variation of $k_{1}$ on $\theta(\eta)$ and $\phi(\eta)$, while that of $\gamma$ on $\theta(\eta)$ and $\phi(\eta)$.

TABLE 7: Analysis on the basis of number of boundary conditions evaluated of the solver for Sisko nano fluid model.

\begin{tabular}{lcccc}
\hline SCENARIOS & Case-1 & Case-2 & Case-3 & Case-4 \\
\hline 1 & 97 & 98 & 98 & 98 \\
2 & 98 & 98 & 98 & 98 \\
3 & 98 & 98 & 98 & 98 \\
4 & 98 & 98 & 98 & 98 \\
5 & 98 & 98 & 98 & 98 \\
6 & 98 & 98 & 97 & 98 \\
7 & 118 & 98 & 98 & 98 \\
8 & 97 & 98 & 98 & 98 \\
9 & 98 & 98 & 98 & 98 \\
10 & 98 & 98 & 98 & 98 \\
11 & 97 & 98 & 98 & 98 \\
13 & 98 & 98 & 98 \\
\hline
\end{tabular}




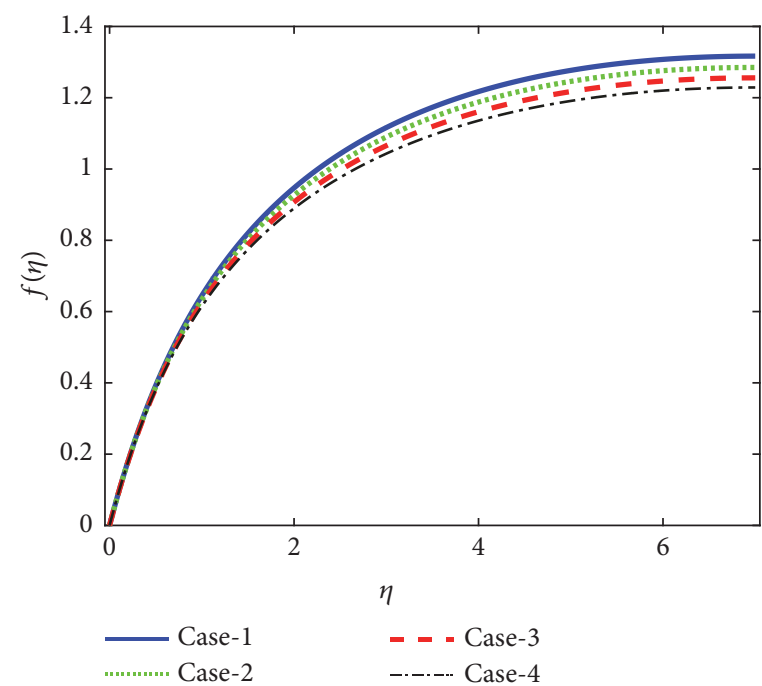

(a) Effect on $f(\eta)$ by varying $\lambda$

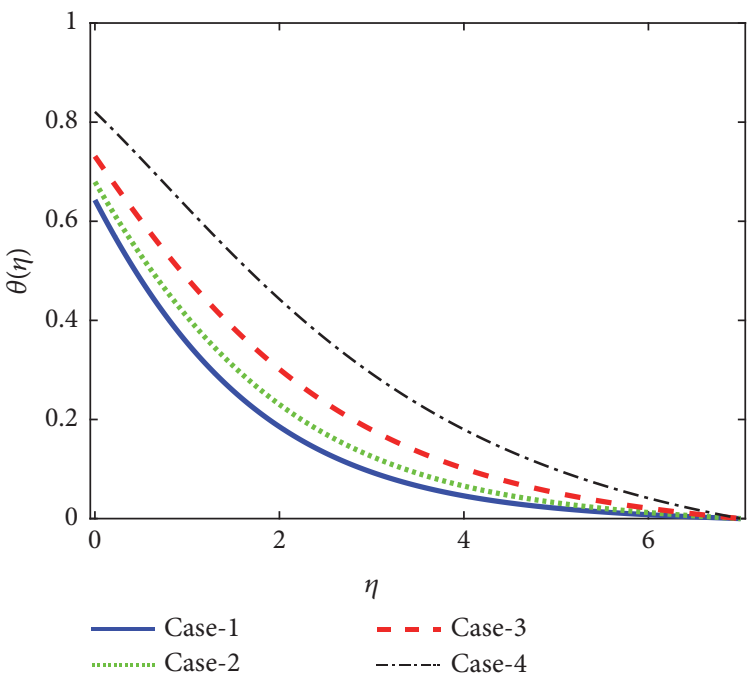

(c) Effect on $\theta(\eta)$ by varying $\lambda$

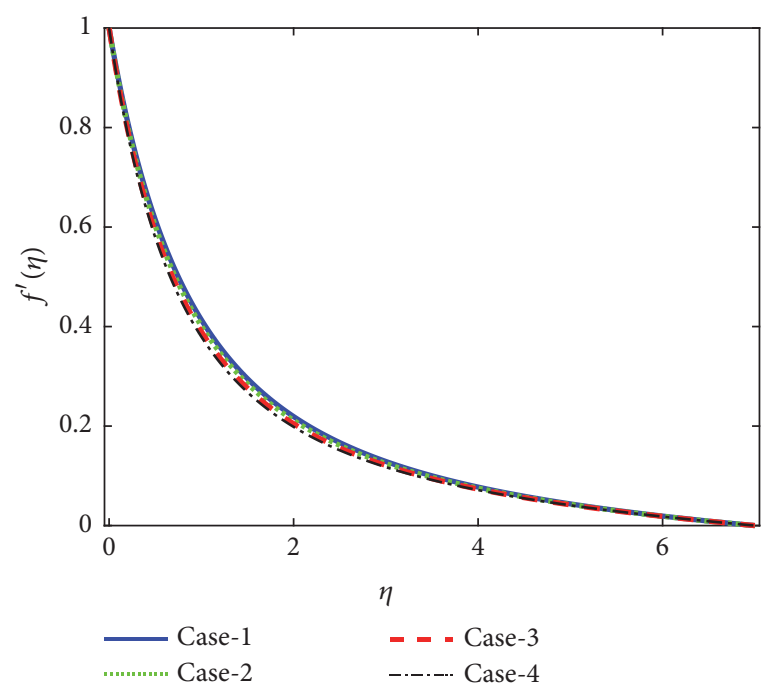

(b) Effect on $f^{\prime}(\eta)$ by varying $\lambda$

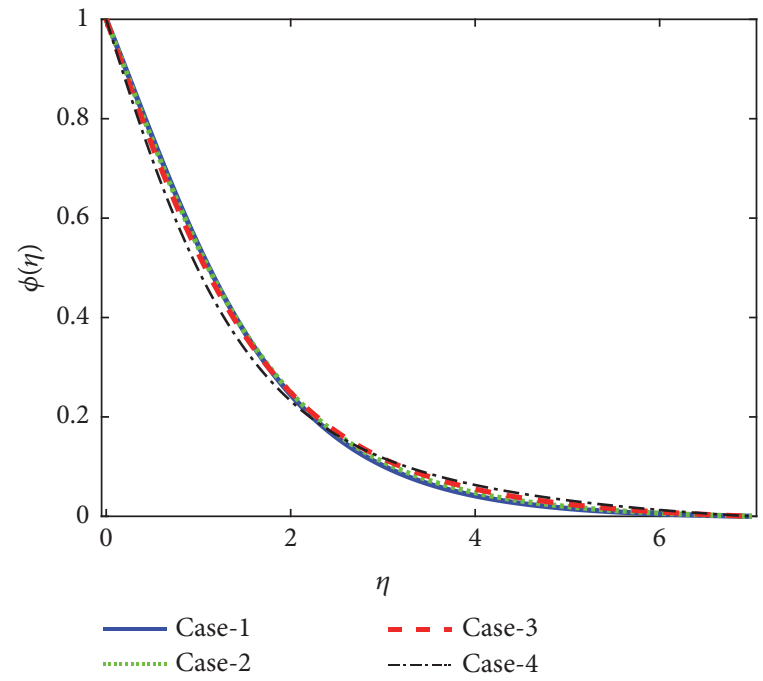

(d) Effect on $\phi(\eta)$ by varying $\lambda$

FiguRE 9: Variation of $\lambda$ on $f(\eta), f^{\prime}(\eta), \theta(\eta)$, and $\phi(\eta)$.

scenario 2, variation of $n$; scenario 3, variation of Pr; scenario 4, variation of Le; scenario 5, variation of $\mathrm{Nb}$; scenario 6, variation of $\mathrm{Nt}$; scenario 7, variation of A; Scenario 8, variation of s; scenario 9, variation of $k_{1}$; scenario 10 , variation of $\gamma$; scenario 11 , variation of $\lambda$; scenario 12 , variation of $R d$; and scenario 13, variation of $\theta_{w}$. Table 2 shows skin friction $-f^{\prime \prime}(0)$ coefficients for various scenarios. Table 3 shows numerical values for surface heat transfer rate $-\theta^{\prime}(0)$ in different scenarios.

Error and complexity analysis of Lobatto IIIA solver is presented in Tables 4-7. Table 4 explains the number of meshes for each case of different scenarios. Table 5 provides maximum error for each case of different scenarios, which show that reasonable accuracy attained by the scheme. Table 6 depicts the number of ODEs evaluated for each case of various scenarios. Table 7 explains the number of boundary conditions (BCs) evaluated during each case for different scenarios. The presented complexity indices can be used for comparison of the given scheme with future similar studies for the Sisko nanofluidic model.

\section{Conclusions}

Characteristics of radiated Sisko nanofluid are studied. Main features of the present analysis are listed below:

(i) The parameter A increases $f(\eta)$ and $f^{\prime}(\eta)$ while decreasing $\theta(\eta)$ and $\phi(\eta)$.

(ii) Velocity profile $f^{\prime}(\eta)$ decreases while concentration profile $\phi(\eta)$ increases with enhancement in s.

(iii) An increase in $k_{1}$ shows decrease in $f(\eta)$ and $f^{\prime}(\eta)$ while showing increase in $\theta(\eta)$ and $\phi(\eta)$.

(iv) Enhancement is observed in $\theta(\eta)$ and $\phi(\eta)$ for higher $\gamma$. 


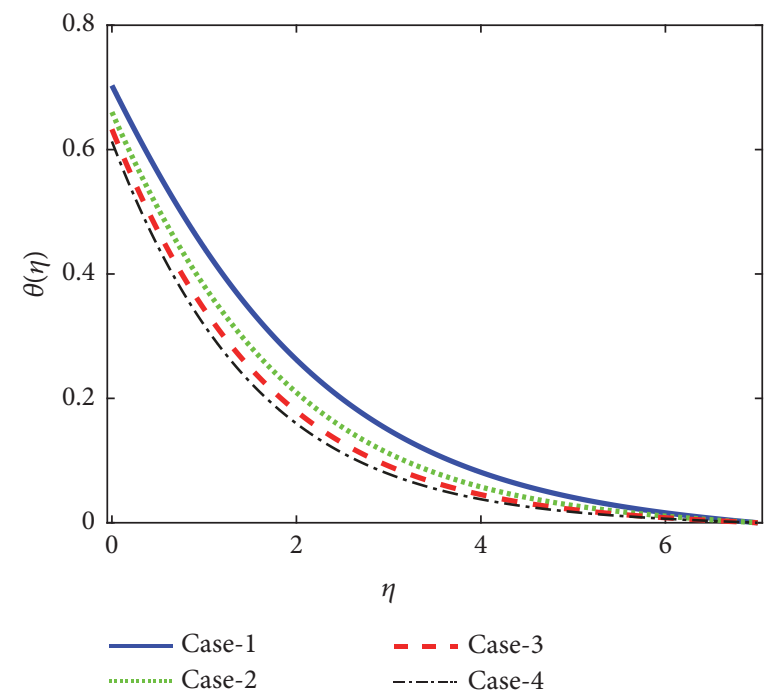

(a) Effect on $\theta(\eta)$ by varying Rd

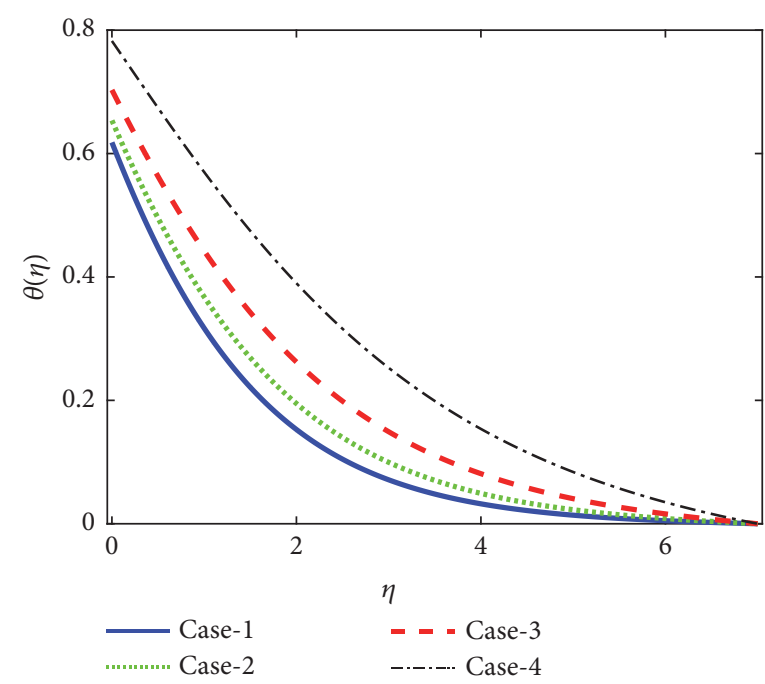

(c) Effect on $\theta(\eta)$ by varying $\theta_{w}$

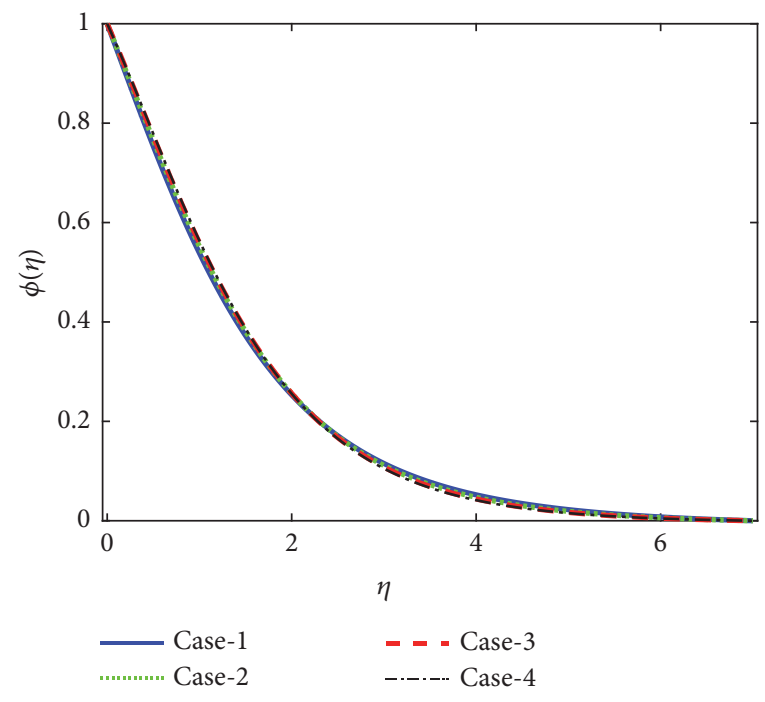

(b) Effect on $\phi(\eta)$ by varying Rd

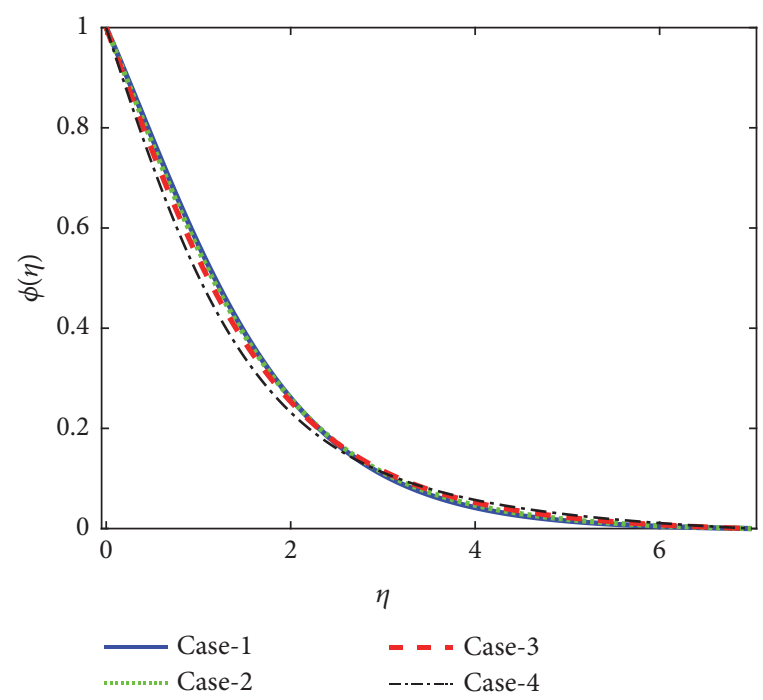

(d) Effect on $\phi(\eta)$ by varying $\theta_{w}$

Figure 10: Variation of $\operatorname{Rd}$ on $\theta$ and $\phi$ while that of $\theta_{w}$ on $\theta(\eta)$ and $\varphi(\eta)$.

(v) A decrease in $f(\eta)$ and $f^{\prime}(\eta)$ while increasing behavior is observed for higher values of $\lambda$.

(vi) Skin friction enhances for higher values of $F r, s, k_{1}$, and $\lambda$ while diminishing for higher values of $n$ and $A$.

(vii) Higher values of $F r, \operatorname{Pr}, L e, N b, N t, s, k 1, \lambda$, and $\theta_{w}$ decay the heat transfer rate while reverse behavior is observed for $n, A, \gamma$, and $R d$.

\section{Nomenclature}

K: Porosity parameter

$k$ : Thermal conductivity

F: Inertia coefficient

$\sigma^{*}$ : Stefan-Boltzmann parameter

$k^{*}$ : Mean absorption parameter

A: Material parameter $q_{w}: \quad$ Heat flux

Pr: Prandtl number

$R d$ : Radiation parameter

Fr: $\quad$ Froude number

Le: Lewis number

Nt: Thermophoresis number

$\mathrm{Nb}$ : Brownian motion parameter

$f: \quad$ Velocity profile parameter

$\theta: \quad$ Temperature profile parameter

$\phi$ : $\quad$ Concentration profile parameter

$k_{1}$ : Permeability parameter

$\lambda: \quad$ Heat generation/absorption variable

$s: \quad$ Stretching rate of surface

$n$ : $\quad$ Fitted rate constant

$\theta_{w}: \quad$ Temperature ratio parameter

$\gamma: \quad$ Biot number

ODE: Ordinary differential equation 
PDE: Partial differential equation

MHD: MagnetoHydroDynamics.

\section{Data Availability}

All the data used in the research is available within the article.

\section{Ethical Approval}

All the authors of the manuscript declared that there is no research involving human participants and/or animal.

\section{Consent}

All the authors of the manuscript declared that there is no material that required informed consent.

\section{Disclosure}

Authors are responsible for any fee if any associated with this manuscript.

\section{Conflicts of Interest}

All the authors of the manuscript declared that there are no potential conflicts of interest.

\section{References}

[1] F. A. L. Dullien, Porous Media: Fluid Transport and Pore Structure, Academic Press, San Diego, USA, 1992.

[2] D. A. Nield and A. Bejan, Convection in Porous Media, Springer, New York, NY, USA, 1999.

[3] H. J. Xu, Z. B. Xing, F. Q. Wang, and Z. M. Cheng, "Review on heat conduction, heat convection, thermal radiation and phase change heat transfer of nanofluids in porous media: Fundamentals and applications," Chemical Engineering Science, 2018.

[4] G. E. Karniadakis, N. Aluru, and A. Beskok, Micro and Nano Flows: Fundamentals and Simulation, 2005.

[5] P. Forchheimer, "Wasserbewegung durch Boden," Zeitschrift des Vereines Deutscher Ingenieure, vol. 45, pp. 1782-1788, 1901.

[6] M. Muskat, The Flow of Homogeneous Fluid through Porous Media, J. W. Edwards, Inc, Ann Arbor, Mich, USA, 6 edition, 1946.

[7] D. Pal and H. Mondal, "Hydromagnetic convective diffusion of species in Darcy-Forchheimer porous medium with nonuniform heat source/sink and variable viscosity," International Communications in Heat and Mass Transfer, vol. 39, no. 7, pp. 913-917, 2012.

[8] N. Vishnu Ganesh, A. K. Abdul Hakeem, and B. Ganga, "Darcy-Forchheimer flow of hydromagnetic nanofluid over a stretching/shrinking sheet in a thermally stratified porous medium with second order slip, viscous and Ohmic dissipations effects," Ain Shams Engineering Journal, 2016.

[9] T. Latif, N. Alvi, Q. Hussain, and S. Asghar, "Peristaltic flow of nonconstant viscosity fluid with nonlinear thermal radiation," Journal of Computational and Theoretical Nanoscience, vol. 14, no. 6, pp. 2681-2693, 2017.

[10] T. Hayat, Z. Nisar, H. Yasmin, and A. Alsaedi, "Peristaltic transport of nanofluid in a compliant wall channel with convective conditions and thermal radiation," Journal of Molecular Liquids, vol. 220, pp. 448-453, 2016.

[11] D. Pal, "Combined effects of non-uniform heat source/sink and thermal radiation on heat transfer over an unsteady stretching permeable surface," Communications in Nonlinear Science and Numerical Simulation, vol. 16, no. 4, pp. 1890-1904, 2011.

[12] A. K. Pandey and M. Kumar, "Natural convection and thermal radiation influence on nanofluid flow over a stretching cylinder in a porous medium with viscous dissipation," Alexandria Engineering Journal, vol. 56, no. 1, pp. 55-62, 2017.

[13] C.-J. Huang, "Lateral mass flux and thermal radiation on natural convection heat and mass transfer from a vertical flat plate in porous media considering Soret/Dufour effects, Lateral mass flux and thermal radiation," Journal of King Saud University Science, vol. 29, no. 2, pp. 241-249, 2017.

[14] M. Kothandapani and J. Prakash, "Effects of thermal radiation parameter and magnetic field on the peristaltic motion of Williamson nanofluids in a tapered asymmetric channel," International Journal of Heat and Mass Transfer, vol. 81, pp. 234245, 2015.

[15] S. Gupta, D. Kumar, and J. Singh, "MHD mixed convective stagnation point flow and heat transfer of an incompressible nanofluid over an inclined stretching sheet with chemical reaction and radiation," International Journal of Heat and Mass Transfer, vol. 118, pp. 378-387, 2018.

[16] M. Sajid, S. A. Iqbal, M. Naveed, and Z. Abbas, "Effect of homogeneous-heterogeneous reactions and magnetohydrodynamics on $\mathrm{Fe} 3 \mathrm{O} 4$ nanofluid for the Blasius flow with thermal radiations," Journal of Molecular Liquids, vol. 233, pp. 115-121, 2017.

[17] S. Qayyum, M. I. Khan, T. Hayat, and A. Alsaedi, "A framework for nonlinear thermal radiation and homogeneousheterogeneous reactions flow based on silver-water and copperwater nanoparticles: A numerical model for probable error," Results in Physics, vol. 7, pp. 1907-1914, 2017.

[18] T. Hayat, S. A. Shehzad, M. Qasim, and S. Obaidat, "Radiative flow of Jeffery fluid in a porous medium with power law heat flux and heat source," Nuclear Engineering and Design, vol. 243, pp. 15-19, 2012.

[19] H. Moghadasi, N. Malekian, M. Bidabadi, and H. Rasam, "Analytical modeling of counterflow non-premixed organic particles combustion: thermal radiation effects," Fuel Processing Technology, vol. 185, pp. 139-150, 2019.

[20] A. S. Dogonchi, M. Waqas, S. M. Seyyedi, M. HashemiTilehnoee, and D. D. Ganji, "CVFEM analysis for Fe3O4-H2O nanofluid in an annulus subject to thermal radiation," International Journal of Heat and Mass Transfer, vol. 132, pp. 473-483, 2019.

[21] K. Zhou and X. Wang, "Thermal radiation modelling of pool fire with consideration on the nonuniform temperature in flame volume," International Journal of Thermal Sciences, vol. 138, pp. 12-23, 2019.

[22] V. V. Svetsov and V. V. Shuvalov, "Thermal radiation and luminous efficiency of superbolides," Earth and Planetary Science Letters, vol. 503, pp. 10-16, 2018.

[23] A. Dehbi, S. Kelm, J. Kalilainen, and H. Mueller, "The influence of thermal radiation on the free convection inside enclosures," Nuclear Engineering and Design, vol. 341, pp. 176-185, 2019.

[24] A. Mushtaq, M. Mustafa, T. Hayat, and A. Alsaedi, "Nonlinear radiative heat transfer in the flow of nanofluid due to solar energy: A numerical study," Journal of the Taiwan Institute of Chemical Engineers, vol. 45, no. 4, pp. 1176-1183, 2014. 
[25] M. Monica and J. Sucharitha, "Effects of nonlinear thermal radiation, non-uniform heat source/sink on MHD stagnation point flow of a casson fluid over a nonlinear stretching sheet with slip conditions," Journal of Nanofluids, vol. 6, no. 4, pp. 692-701, 2017.

[26] B. Mahanthesh, B. J. Gireesha, and R. S. R. Gorla, "Nonlinear radiative heat transfer in MHD three-dimensional flow of water based nanofluid over a non-linearly stretching sheet with convective boundary condition," Journal of the Nigerian Mathematical Society, vol. 35, no. 1, pp. 178-198, 2016.

[27] M. M. Bhatti, A. Zeeshan, and R. Ellahi, "Study of heat transfer with nonlinear thermal radiation on sinusoidal motion of magnetic solid particles in a dusty fluid," Journal of Theoretical and Applied Mechanics, vol. 46, no. 3, pp. 75-94, 2016.

[28] N. S. Akbar, S. Nadeem, R. Ul Haq, and Z. H. Khan, "Radiation effects on MHD stagnation point flow of nano fluid towards a stretching surface with convective boundary condition," Chinese Journal of Aeronautics, vol. 26, no. 6, pp. 1389-1397, 2013.

[29] T. Hayat, M. I. Khan, S. Qayyum, A. Alsaedi, and M. I. Khan, "New thermodynamics of entropy generation minimization with nonlinear thermal radiation and nanomaterials," Physics Letters A, vol. 382, no. 11, pp. 749-760, 2018.

[30] M. Rashid, M. I. Khan, T. Hayat, and A. Alsaedi, "Entropy generation in flow of ferromagnetic liquid with nonlinear radiation and slip condition," Journal of Molecular Liquids, vol. 276, pp. 441-452, 2019.

[31] M. W. A. Khan, M. I. Khan, T. Hayat, and A. Alsaedi, "Entropy generation minimization (EGM) of nanofluid flow by a thin moving needle with nonlinear thermal radiation," Physica B: Condensed Matter, vol. 534, pp. 113-119, 2018.

[32] R. Kumar, M. Sheikholeslami, and A. J. Chamkha, "Irreversibility analysis of the three dimensional flow of carbon nanotubes due to nonlinear thermal radiation and quartic chemical reactions," Journal of Molecular Liquids, vol. 274, pp. 379-392, 2019.

[33] F. A. Soomro, M. Usman, R. Ul Haq, and W. Wang, "Melting heat transfer analysis of Sisko fluid over a moving surface with nonlinear thermal radiation via Collocation method," International Journal of Heat and Mass Transfer, vol. 126, pp. 1034-1042, 2018.

[34] Z. Li, M. Sheikholeslami, A. Shafee, S. Saleem, and A. J. Chamkha, "Effect of dispersing nanoparticles on solidification process in existence of Lorenz forces in a permeable media," Journal of Molecular Liquids, vol. 266, pp. 181-193, 2018.

[35] M. A. Sadiq, A. U. Khan, S. Saleem, and S. Nadeem, "Numerical simulation of oscillatory oblique stagnation point flow of a magneto micropolar nanofluid," RSC Advances, vol. 9, no. 9, pp. 4751-4764, 2019.

[36] S. Saleem, H. Firdous, S. Nadeem, and A. U. Khan, "Convective heat and mass transfer in magneto walter's B nanofluid flow induced by a rotating Cone," Journal for Science and Engineering, vol. 44, no. 2, pp. 1515-1523, 2019.

[37] S. Saleem, S. Nadeem, M. M. Rashidi, and C. S. K. Raju, "An optimal analysis of radiated nanomaterial flow with viscous dissipation and heat source," Microsystem Technologies, vol. 25, no. 2, pp. 683-689, 2019.

[38] M. R. Eid and K. L. Mahny, "Flow and heat transfer in a porous medium saturated with a sisko nanofluid over a nonlinearly stretching sheet with heat generation/absorption," Heat Transfer - Asian Research, vol. 47, no. 1, pp. 54-71, 2018.

[39] T. Sajid, M. Sagheer, S. Hussain, and M. Bilal, "DarcyForchheimer flow of Maxwell nanofluid flow with nonlinear thermal radiation and activation energy," AIP Advances, vol. 8, no. 3, Article ID 035102, 2018.

[40] L. F. Shampine, J. Kierzenka, and M. W. Reichelt, "Solving boundary value problems for ordinary differential equations in MATLAB with bvp4c," Tutorial Notes, 2000, pp. 1-27, 2000. 


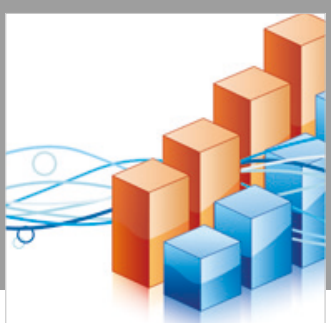

Advances in

Operations Research

\section{-n-m}
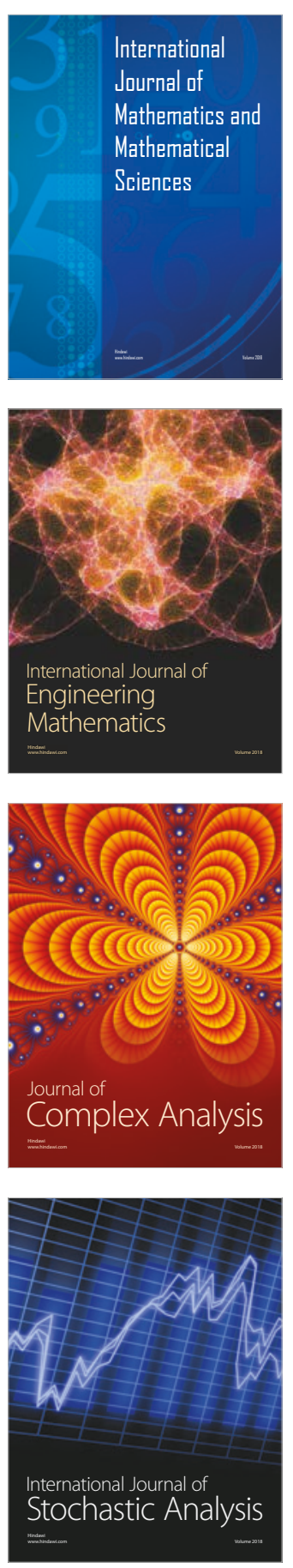
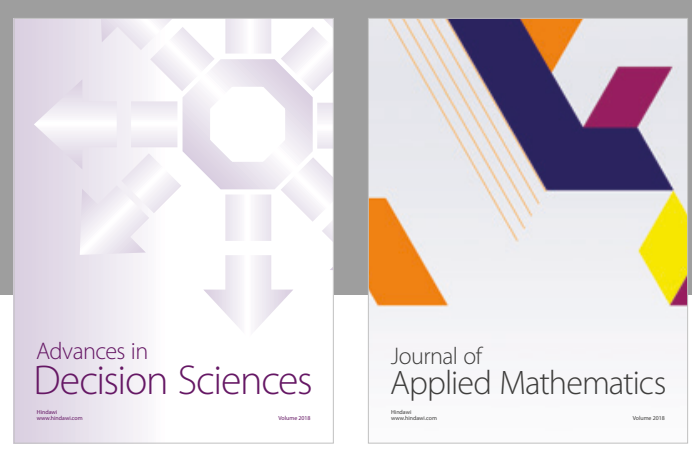

Journal of

Applied Mathematics
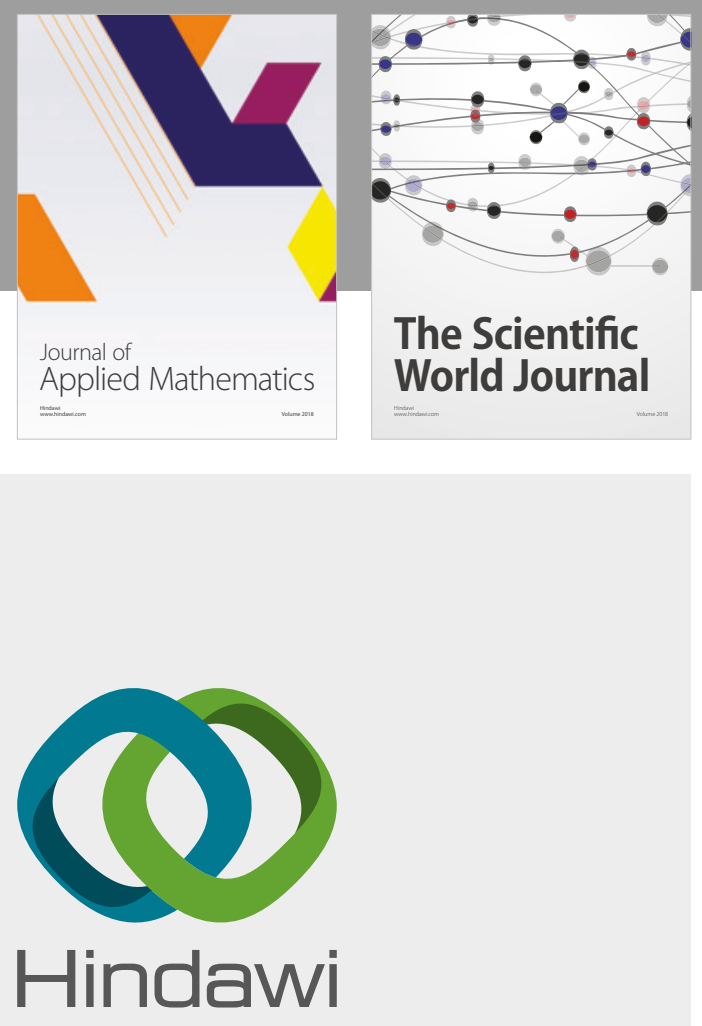

Submit your manuscripts at

www.hindawi.com

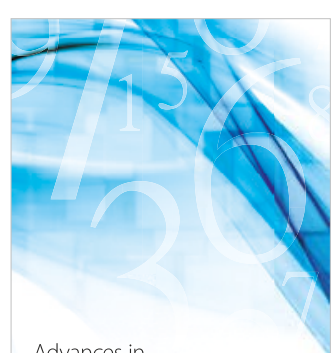

Advances in
Numerical Analysis
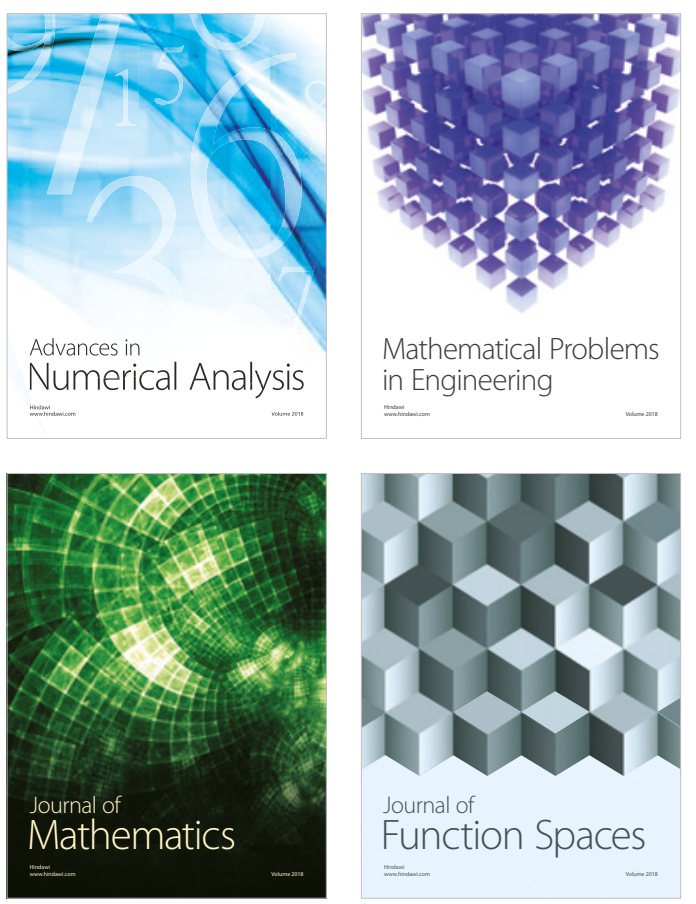

Mathematical Problems in Engineering

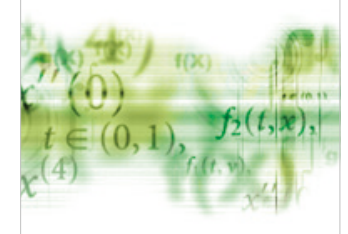

International Journal of

Differential Equations

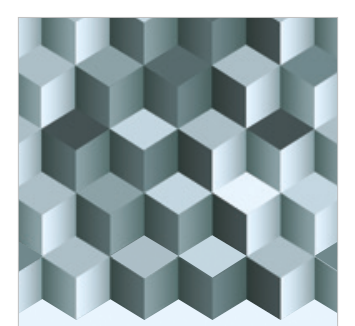

Journal of

Function Spaces

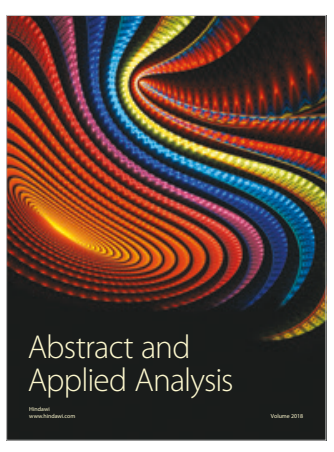

The Scientific

World Journal

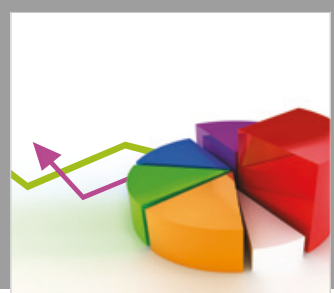

Journal of

Probability and Statistics
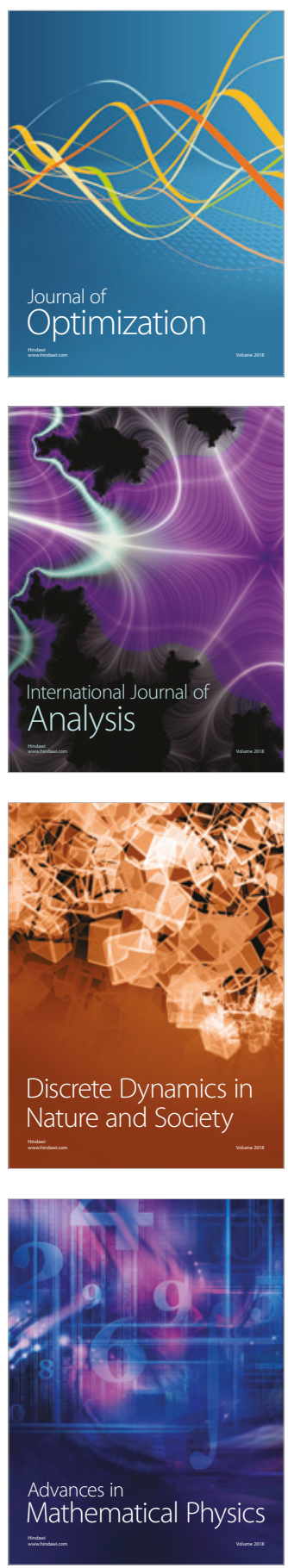